\title{
LA FAMILIA ASTERACEAE EN EL ESTADO DE NAYARIT (MEXICO)
}

\author{
EnRIQUe Ortiz-Bermudez \\ Jose LUIS VILLASEÑOR \\ Y \\ Oswaldo Tellez \\ Instituto de Biología, UNAM \\ Departamento de Botánica \\ Apartado postal 70-367 \\ 04510 México, D.F.
}

\begin{abstract}
RESUMEN
El estado de Nayarit se ubica en una posición geográfica estratégica al encontrarse en el vértice de tres importantes cadenas montañosas del país (Eje Volcánico Transversal, Sierra Madre Occidental y Sierra Madre del Sur), lo que determina en gran medida su alta riqueza florística. En la entidad, la familia Asteraceae, representada por 122 géneros y 447 especies, es la más diversa de todas las fanerógamas. En este trabajo se presenta una lista actualizada de las especies de Asteraceae; asimismo, se compara su diversidad con la de otros estados vecinos. Se presenta igualmente una lista de las especies endémicas a Nayarit.
\end{abstract}

\section{ABSTRACT}

The state of Nayarit occupies a strategic position in Mexico; it encompasses the vertex of three important mountain ranges (Eje Volcánico Transversal, Sierra Madre Occidental and Sierra Madre del Sur); this largely determines its high floristic diversity. In the state, Asteraceae is the most diverse family of flowering plants, with 122 genera and 447 species. This paper includes an updated list of species of Asteraceae found in Nayarit and this diversity is compared to that found in the neighbouring states. A list of the endemic species to Nayarit is also included.

\section{INTRODUCCION}

La familia Asteraceae Dumort. (=Compositae Giseke) es de distribución prácticamente cosmopolita y es una de las más comunes en cualquier tipo de habitat. México es uno de sus principales centros de diversificación y en su territorio se encuentra la concentración más cuantiosa de géneros y especies de todo el mundo. En el estado de Nayarit la familia constituye el grupo taxonómico más importante, seguido de Fabaceae y Poaceae (Téllez, 1995). 
Aunque la familia es una de las mejor conocidas dentro de la flora nacional, aún se tiene un pobre conocimiento de ella a nivel regional o estatal. Existen entidades políticas que todavía carecen de listados florísticos y en consecuencia de un recuento actualizado del número de especies de Asteraceae que contienen. Entre ellos se cuenta Nayarit, el cual hasta fechas recientes no había sido objeto de estudio florístico detallado (Téllez, 1995).

El objetivo de este trabajo es presentar una lista actualizada de las especies de Asteraceae que forman parte de la flora de Nayarit y comparar esta riqueza con la que se presenta en los estados vecinos.

\section{Area de estudio}

Nayarit está situado en la porción centro-occidental de la República Mexicana (Fig. 1). Con una extensión de $27,865 \mathrm{~km}^{2}$, cubre $1.4 \%$ de la República Mexicana e incluye el archipiélago de las Islas Marías, que se ubica en el Océano Pacífico, a 110 km del Puerto de San Blas (Anónimo, 1981; Tamayo, 1962).

El estado presenta un relieve muy accidentado y su compleja topografía está determinada por la presencia de tres de los principales complejos montañosos del país: el extremo sur de la Sierra Madre Occidental, el extremo occidental del Eje Volcánico Transversal y el extremo norte de la Sierra Madre del Sur. Su territorio también incluye el extremo sur de la Planice Costera Noroccidental (Anónimo, 1981; Rzedowski, 1978). La Sierra Madre Occidental se ubica de noroeste a sureste y corre paralela al Océano Pacífico; presenta altitudes máximas entre 2000-2750 m. El Eje Volcánico Transversal aporta importantes elementos a la topografía del estado, como son los volcanes San Juan, Ceboruco, Vigía, Sangangüey, San Pedro Lagunillas y Tepetiltic, con alturas entre 20002700 m s.n.m. (De la Cerda et al., 1989). Por su parte, la Sierra Madre del Sur contribuye con serranías de tipo calizo, como la de Sierra Vallejo. Finalmente la Llanura Costera del Pacífico o Planicie Costera Noroccidental se extiende del norte de Sonora hasta el centro de Nayarit (De la Cerda et al., 1989) y comprende una gran porción de este último (Anónimo, 1981); su topografía es plana o con elevaciones que no rebasan los $100 \mathrm{~m}$ de altitud en promedio, pero existen prominencias que alcanzan los $300 \mathrm{~m}$ hacia la zona del Río San Pedro.

La mayoría de las rocas de Nayarit son ígneas, extrusivas e intrusivas (Terciario). Le siguen en importancia los depósitos aluviales, lacustres, palustres y litorales, los cuales datan del Cuaternario. En menor proporción se encuentran los depósitos sedimentarios clásticos del Terciario y afloramientos de rocas sedimentarias marinas del Mesozoico (Triásico y Cretácico) (Anónimo, 1981; Ferrusquía-Villafranca, 1993).

Los principales tipos de clima que se presentan en Nayarit son tres, A, B y C (García, 1988). Los del tipo A son tropicales lluviosos, con temperatura media del mes más frío superior a los $18^{\circ}$; los del tipo B son secos, mientras que los del C son templados lluviosos. El subtipo más común es el Aw (caliente subhúmedo con lluvias en verano), que cubre $67 \%$ de la superficie del estado y se entremezcla con el Am. El subtipo Am (caliente húmedo con lluvias en verano) abarca $0.4 \%$ del estado. El subtipo $A(C)$ se encuentra hacia la parte central de la Sierra Madre Occidental. Los climas del tipo B, los más secos, están escasamente representados en Nayarit y se encuentran hacia la porción sur y suroccidental, en los límites con el estado de Jalisco. Los del tipo $\mathrm{C}$ se localizan en las partes altas del Eje Volcánico Transversal y la Sierra Madre Occidental; ocupan 9\% del territorio estatal. 


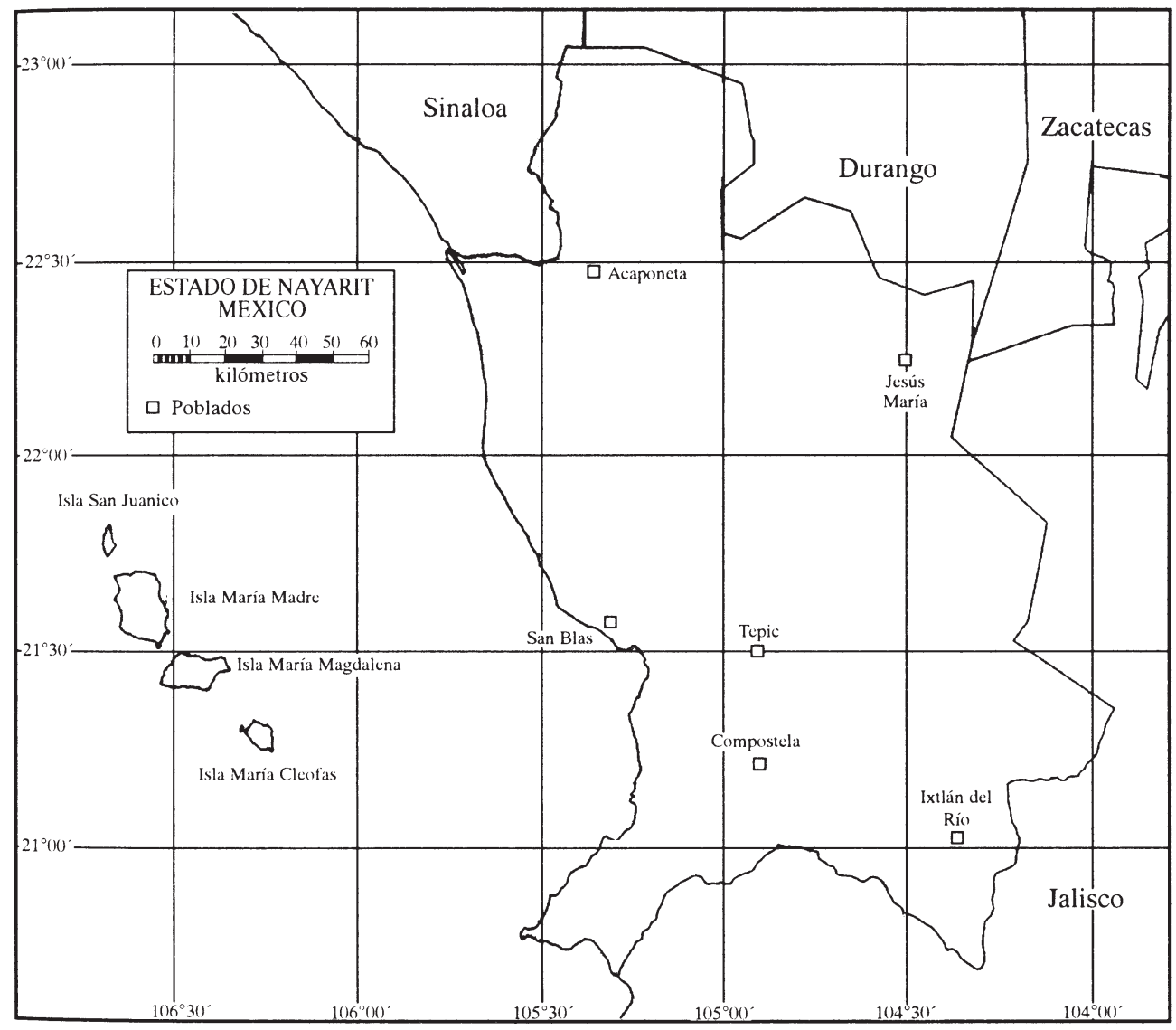

Fig. 1. Mapa del estado de Nayarit. 
En el estado de Nayarit se han registrado los siguientes tipos de vegetación, de acuerdo con la clasificación de Rzedowski (1978): bosque tropical caducifolio, bosque tropical subcaducifolio, bosque de Byrsonima y Curatella, bosque de Quercus, bosque de Pinus, bosque de Pinus-Quercus, bosque mesófilo de montaña, palmar, vegetación halófila, vegetación riparia, así como diversas asociaciones de vegetación acuática (manglar, carrizal, tular, etc.) y las asociaciones secundarias.

\section{MATERIALES Y METODOS}

Con el objeto de integrar la lista de especies se llevó a cabo una revisión exhaustiva de la literatura florístico-taxonómica. Se consultó también el material depositado en varios herbarios de México y del extranjero, pero principalmente el incluido en el Herbario Nacional del Instituto de Biología, de la Universidad Nacional Autónoma de México (MEXU).

Gran parte de los ejemplares citados son producto de más de 25 expediciones de recolecta realizadas en el estado como parte del proyecto "Flora de Nayarit" (Téllez, 1995). Todo el material de referencia registrado en el apéndice se encuentra debidamente montado e incorporado en los herbarios citados.

\section{RESULTADOS}

Las Asteraceae en Nayarit suman hasta la fecha 447 especies, distribuidas en 122 géneros (ver Apéndice). Dicha cantidad indica que el estado es importante, florísticamente hablando, por la gran cantidad de miembros de la familia que alberga. Su importancia puede enfatizarse si se compara dicha diversidad con la de otros estados de la región. En el Cuadro 1 se indican las cifras de géneros y especies conocidas de los estados vecinos a Nayarit y se muestra que este último se encuentra entre las entidades con mayor riqueza de Asteraceae, no obstante que cuenta con una menor superficie.

Cuadro 1. Géneros, especies y taxa infraespecíficos registrados para Nayarit y estados vecinos.

\begin{tabular}{|lccc|}
\hline Estado & Géneros & Especies & Taxa infraespecíficos \\
\hline Nayarit & 122 & 447 & 24 \\
Durango & 149 & 602 & 38 \\
Jalisco & 157 & 750 & 55 \\
Sinaloa & 114 & 387 & 31 \\
Zacatecas & 132 & 377 & 22 \\
\hline
\end{tabular}

En su territorio se registran 15 especies endémicas (Cuadro 2), un número relativamente alto si se considera la escasa extensión geográfica. Tomando como referencia una comarca más amplia que incluye a los estados que limitan con Nayarit (ver Cuadro 3), 
Cuadro 2. Especies de Asteraceae endémicas a Nayarit.

Acourtia nudiuscula (B.L. Rob.) B.L. Turner

Encelia angustifolia Greenm.

Lasianthaea rosei (Greenm.) McVaugh

Psacalium filicifolium (Rydb.) H. Rob. \& Brettell

Sinclairia gentryi (H. Rob.) B.L. Turner

Smallanthus apus (S.F. Blake) H. Rob.

Verbesina fusiformis McVaugh

Verbesina hispida McVaugh

Verbesina madrensis Greenm.

Verbesina nayaritensis B.L. Turner

Verbesina rosei B.L. Rob. \& Greenm.

Vernonia feddemae McVaugh

Viguiera huajicoria B.L. Turner

Viguiera kingii McVaugh

Wedelia simsioides McVaugh

el número de endemismos se incrementa a 62 , lo que representa $13.9 \%$ del total estatal. En el Cuadro 3 se indican los patrones de distribución correspondientes a estos elementos de distribución restringida.

Cuadro 3. Distribución de las especies endémicas a Nayarit y estados vecinos. $1=$ Nayarit; $2=$ Nayarit, Durango; $3=$ Nayarit, Jalisco; $4=$ Nayarit, Sinaloa; $5=$ Nayarit, Durango, Jalisco; $6=$ Nayarit, Durango, Jalisco, Sinaloa; $7=$ Nayarit, Durango, Jalisco, Zacatecas; $8=$ Nayarit, Jalisco, Sinaloa; $9=$ Nayarit, Jalisco, Zacatecas.

\begin{tabular}{|c|c|c|c|c|c|c|c|c|c|}
\hline Especies endémicas & 1 & 2 & 3 & 4 & 5 & 6 & 7 & 8 & 9 \\
\hline Acourtia longifolia & & & $X$ & & & & & & \\
\hline Acourtia macrocephala & & $x$ & & & & & & & \\
\hline Acourtia nudiuscula & $x$ & & & & & & & & \\
\hline Ageratina beamanii & & & & & $\mathrm{x}$ & & & & \\
\hline Ageratina grashoffii & & $x$ & & & & & & & \\
\hline Ageratina jaliscensis & & & $x$ & & & & & & \\
\hline Ageratina leptodictyon & & & & & & & & & $x$ \\
\hline Axiniphyllum durangense & & & & & $x$ & & & & \\
\hline Bidens cordylocarpa & & & $x$ & & & & & & \\
\hline Brickellia cuspidata & & & $x$ & & & & & & \\
\hline Brickellia magnifica & & & $x$ & & & & & & \\
\hline Brickellia worthingtonii & & & & & $x$ & & & & \\
\hline Coreopsis cuneifolia & & & & & $\mathrm{x}$ & & & & \\
\hline Coreopsis rudis & & & & & & & $x$ & & \\
\hline Encelia angustifolia & $x$ & & & & & & & & \\
\hline Erigeron exilis & & & $x$ & & & & & & \\
\hline Eupatorium trinervium & & & & & & $x$ & & & \\
\hline
\end{tabular}


Acta Botánica Mexicana (1998), 44: 25-57

Cuadro 3. Continuación.

\begin{tabular}{|c|c|c|c|c|c|c|c|c|c|}
\hline Especies endémicas & 1 & 2 & 3 & 4 & 5 & 6 & 7 & 8 & 9 \\
\hline Guardiola carinata & & & $x$ & & & & & & \\
\hline Heliopsis novogaliciana & & & & & & $x$ & & & \\
\hline Hofmeisteria mexiae & & & $\mathrm{X}$ & & & & & & \\
\hline Lasianthaea rosei & $x$ & & & & & & & & \\
\hline Melampodium rosei & & & & & & $x$ & & & \\
\hline Packera rosei & & & & & $x$ & & & & \\
\hline Perityle trichodonta & & & & & & & & & $x$ \\
\hline Perymenium uxoris & & & $x$ & & & & & & \\
\hline Pinaropappus diguetii & & & & & & & & & $x$ \\
\hline Psacalium cervinum & & & $x$ & & & & & & \\
\hline Psacalium eriocarpum & & & $x$ & & & & & & \\
\hline Psacalium filicifolium & $x$ & & & & & & & & \\
\hline Pseudognaphalium jaliscense & & & $x$ & & & & & & \\
\hline Sinclairia cervina & & & & & & & & $x$ & \\
\hline Sinclairia gentryi & $x$ & & & & & & & & \\
\hline Sinclairia pringlei & & & $x$ & & & & & & \\
\hline Sinclairia similis & & & $\mathrm{X}$ & & & & & & \\
\hline Smallanthus apus & $x$ & & & & & & & & \\
\hline Stevia lasioclada & & & & & & & & $x$ & \\
\hline Stevia myricoides & & & & & & & & $x$ & \\
\hline Stevia ovalis & & & $x$ & & & & & & \\
\hline Stevia rosei & & & & & & $x$ & & & \\
\hline Stevia villaregalis & & & & & $x$ & & & & \\
\hline Tagetes hartwegii & & & $x$ & & & & & & \\
\hline Verbesina fusiformis & $x$ & & & & & & & & \\
\hline Verbesina hispida & $x$ & & & & & & & & \\
\hline Verbesina lottiana & & & $x$ & & & & & & \\
\hline Verbesina madrensis & $x$ & & & & & & & & \\
\hline Verbesina nayaritensis & $x$ & & & & & & & & \\
\hline Verbesina rosei & $x$ & & & & & & & & \\
\hline Verbesina tequilana & & & $x$ & & & & & & \\
\hline Verbesina vallartana & & & $x$ & & & & & & \\
\hline Vernonia bolleana & & $x$ & & & & & & & \\
\hline Vernonia feddemae & $x$ & & & & & & & & \\
\hline Viguiera angustifolia & & & & & & $x$ & & & \\
\hline Viguiera huajicoria & $x$ & & & & & & & & \\
\hline Viguiera kingii & $x$ & & & & & & & & \\
\hline Viguiera latibracteata & & & & & & $x$ & & & \\
\hline Viguiera parkinsonii & & & & & & $x$ & & & \\
\hline Viguiera pringlei & & & $x$ & & & & & & \\
\hline Viguiera schultzii & & & $x$ & & & & & & \\
\hline Wedelia aggregata & & & $x$ & & & & & & \\
\hline Wedelia grayi & & & $x$ & & & & & & \\
\hline Wedelia vexata & & & $x$ & & & & & & \\
\hline Zinnia leucoglossa & & & & $x$ & & & & & \\
\hline
\end{tabular}


Como se señaló previamente, Nayarit administra políticamente al archipiélago de las Islas Marías, en las cuales se ha detectado una flora interesante, estrechamente ligada a las regiones tropicales del continente (Lenz, 1995) y con un buen número de endemismos. En el Cuadro 4 se presenta una enumeración de las especies de Asteraceae registradas en las islas.

Cuadro 4. Especies de Asteraceae registradas para las Islas Marías (basado en Lenz, 1995).

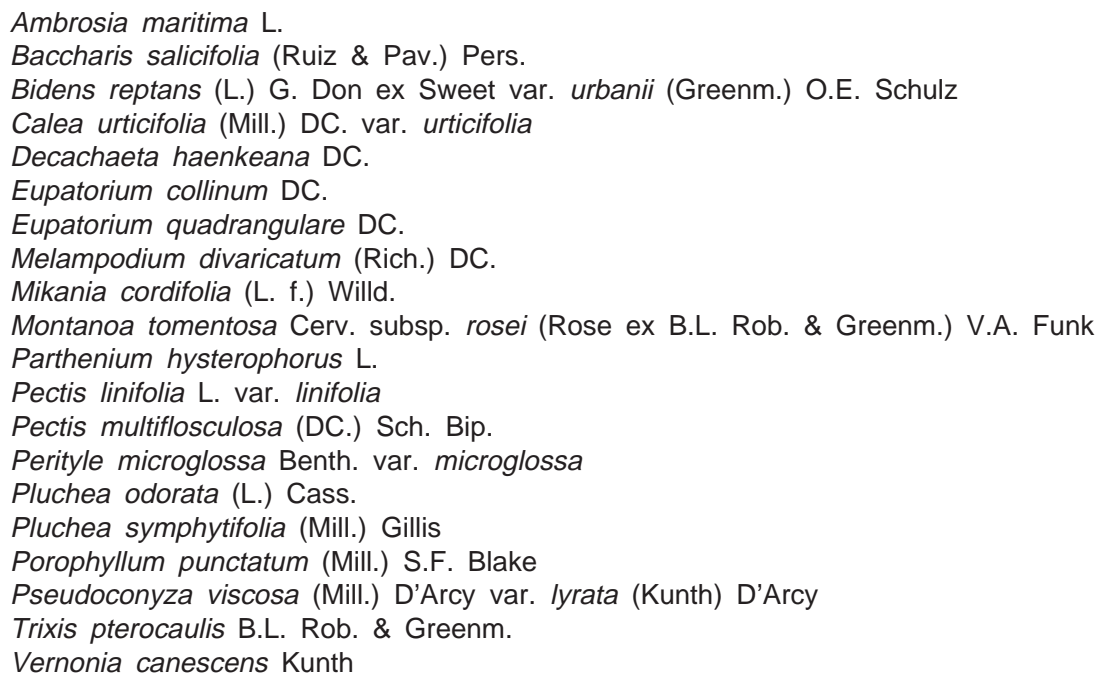

\section{DISCUSION}

El estado de Nayarit, no obstante su pequeña superficie, cuenta con una amplia diversidad de miembros de Asteraceae. Esta riqueza seguramente es el resultado de la combinación de un conjunto de factores bióticos y abióticos, tales como su complicada fisiografía y su posición geográfica estratégica.

La flora del estado representa un mosaico muy interesante de elementos con diferentes afinidades biogeográficas (Téllez, 1995). En particular es interesante la marcada influencia de elementos del Eje Volcánico Transversal y de la Sierra Madre Occidental. El mayor número de endemismos (Cuadro 3) se concentra en esas regiones fisiográficas, que encuentran en el estado sus extremos occidental y meridional respectivamente. Un análisis más detallado de los aspectos biogeográficos y del papel que juega el estado en la conservación de la flora sinanterológica del occidente de México está en proceso de elaboración como un trabajo complementario (Villaseñor et al., en preparación).

El listado que aquí se presenta enriquece substancialmente el conocimiento de una de las regiones más ricas en especies y en endemismos de Asteraceae del país. Asimismo 
complementa, aunque modestamente, la monumental obra que en la región de Nueva Galicia ha emprendido el Dr. Rogers McVaugh (1984).

\section{AGRADECIMIENTOS}

El trabajo de campo para conjuntar este listado fue en gran medida posible gracias al apoyo económico que para el estudio de la flora del estado ha sido otorgado por CONACYT en 1985-1987 (PCCNCNA-031529), 1988-1989 (P218CCOC880112), 19901991 (P218CCOC827792 y D112-903588) y 1993-1994 (0455-N9108), por la National Geographic Society (4349-89) y por la Comisión Nacional para el Conocimiento y Uso de la Biodiversidad (CONABIO-FB069/P083/93).

\section{LITERATURA CITADA}

Anónimo. 1981. Síntesis geográfica de Nayarit. Secretaría de Programación y Presupuesto. México, D.F.

Bierner, M. W. 1972. Taxonomy of Helenium sect. Tetrodus and a conspectus of North American Helenium (Compositae). Brittonia 24: 331-355.

De la Cerda, C. H., E. Ojeda T., A. Santos O. y C. A. Ortiz S. 1989. Provincias, regiones y subregiones terrestres de México. Colegio de Postgraduados. Chapingo, México. 624 pp.

Ferrusquía-Villafranca, I. 1993. Geology of Mexico: A synopsis. In: Ramamoorthy, T. P., R. Bye, J. Fa y A. Lot (eds.). Biological diversity of Mexico: Origins and distribution. Oxford University Press. Nueva York. pp. 3-107.

García, E. 1988. Modificaciones al sistema de clasificación climática de Köppen. 4a. edición. Instituto de Geografía, Universidad Nacional Autónoma de México. México, D.F. 217 pp.

Lenz, L. W. 1995. Plants of the Tres Marias Islands, Nayarit, Mexico. Aliso 14: 19-34.

McVaugh, R. 1984. Compositae. In: Anderson, W. R. (ed.). Flora Novo-Galiciana. Vol. 12. The University of Michigan Press. Ann Arbor. 1157 pp.

Nesom, G. L. 1995. Revision of Chaptalia (Asteraceae: Mutiseae) from North America and continental Central America. Phytologia 78(3): 153-188.

Rzedowski, J. 1978. Vegetación de México. Limusa. México, D.F. 432 pp.

Tamayo, J. L. 1962. Geografía general de México. Geografía física. 2a. ed. Instituto Mexicano de Investigaciones Económicas. México, D.F. Tomo 2. 633 pp.

Téllez V., O. 1995. Flora, vegetación y fitogeografía de Nayarit, México. Tesis de Maestría en Ciencias. Facultad de Ciencias, Universidad Nacional Autónoma de México. México, D.F. 166 pp.

Turner, B. L. 1996. The Comps of Mexico. A systematic account of the family Asteraceae. Vol. 6. Tageteae and Anthemideae. The University of Texas. Plant Resources Center. Austin, Texas. $93 \mathrm{pp}$.

Turner, B. L. 1997. The Comps of Mexico. A systematic account of the Family Asteraceae. Vol. 1. Eupatorieae. The University of Texas. Plant Resources Center. Austin, Texas. 272 pp. 
Ortiz-Bermúdez et al.: La Familia Asteraceae en el Estado de Nayarit

\section{APENDICE}

Lista de especies registradas para el estado de Nayarit y de especímenes que documentan su presencia.

Achillea millefolium L. [Ruenes-Morales 77 (MEXU, UAN)].

Acmella oppositifolia (Lam.) R.K. Jansen var. oppositifolia [Téllez-Valdés 9431 (MEXU)]. Acmella radicans (Jacq.) R.K. Jansen var. radicans [Benítez-Paredes 2997 (MEXU); Benítez-Paredes 3619 (MEXU); Calzada 18818 (ENCB, MEXU, MICH, MO); TéllezValdés 8269 (MEXU); Téllez-Valdés 11146 (ENCB, MEXU, MICH); Téllez-Valdés 11849 (MEXU); Téllez-Valdés 12858 (MEXU); Téllez-Valdés 12863 (MEXU)].

Acourtia fruticosa (Llave) B.L. Turner [Calzada 18827 (MEXU, MICH); Téllez-Valdés 9600 (MEXU); Téllez-Valdés 10133 (MEXU)].

Acourtia grandifolia (S. Watson) Reveal \& R.M. King [Magaña-Rueda 140 (MEXU)].

Acourtia longifolia (S.F. Blake) Reveal \& R.M. King [Palmer 1972 (Citado por McVaugh, 1984); McVaugh 730 (Citado por McVaugh, 1984)].

Acourtia macrocephala Sch. Bip. ex Seem. [Seemann s.n. (K, MICH, GH)].

Acourtia nudiuscula (B.L. Rob.) B.L. Turner [Flores-Franco 2020 (MEXU); Téllez-Valdés 10132 (MEXU)].

Acourtia patens (A. Gray) Reveal \& R.M. King [Flores-Franco 1597 (MEXU); Flores-Franco 1699 (MEXU); Tenorio-Lezama 16398 (MEXU); Tenorio-Lezama 16636 (MEXU); Tenorio-Lezama 16676 (MEXU)].

Acourtia wislizeni (A. Gray) Reveal \& R.M. King var. megacephala (A. Gray) Reveal \& R.M. King [Calzada 19201 (MEXU); Téllez-Valdés 9446 (MEXU); Téllez-Valdés 10146 (MEXU)].

Adenophyllum anomalum (Canby \& Rose) Strother [Téllez-Valdés 8266 (MEXU)]. Adenophyllum porophyllum (Cav.) Hemsl. var. cancellatum (Cass.) Strother [BenítezParedes 3391 (MEXU); Calzada 18837 (MEXU); Flores-Franco 1587 (MEXU); TéllezValdés 9827 (MEXU); Téllez-Valdés 10541 (MEXU); Tenorio-Lezama 16644 (MEXU)]. Adenophyllum porophyllum (Cav.) Hemsl. var. porophyllum [Ferris 5858 (Citado por McVaugh, 1984)].

Adenophyllum squamosum (A. Gray) Strother [Calzada 19200 (ENCB, MEXU, MICH, MO, XAL); Flores-Franco 876 (MEXU); Flores-Franco 1909 (MEXU); Flores-Franco 3403 (ENCB, MEXU, MICH, MO); Téllez-Valdés 10275 (MEXU); Tenorio-Lezama 15619 (MEXU)].

Ageratella microphylla (Sch. Bip.) A. Gray ex S. Watson [Bauml 1102 (MEXU); Calzada 18808 (EBCH, ENCB, MEXU, MICH, MO, US, XAL); Flores-Franco 2468 (MEXU); McVaugh 25759 (MEXU, MICH); Ramírez-Rodríguez 785 (MEXU); Téllez-Valdés 11405 (MEXU); Téllez-Valdés 11430 (MEXU); Téllez-Valdés 11790 (MEXU); Tenorio-Lezama 16700 (MEXU); Tenorio-Lezama 16903 (MEXU)].

Ageratina areolaris (DC.) Gage [Aguilar-Orozco s.n. (MEXU); Blanco-Correa s.n. (MEXU); Boutin 2659 (MEXU); Calzada 19058 (MEXU); Flores-Franco 3166 (ENCB, MEXU, 
MICH, MO, US, XAL); Flores-Franco 3199 (MEXU); Flores-Franco 3261 (MEXU); FloresFranco 4462 (MEXU); Flores-Franco 4513 (MEXU); González-Flores 830 (MEXU, UAN); González-Flores 863 (MEXU, UAN); Téllez-Valdés 9744 (MEXU); Tenorio-Lezama 16803 (MEXU); Tenorio-Lezama 16975 (MEXU)].

Ageratina arsenei (B.L. Rob.) R.M. King \& H. Rob. [Calzada 19226 (ENCB, MEXU, MICH, MO, XAL); Flores-Franco 1891 (MEXU); Flores-Franco 2539 (MEXU); Flores-Franco 3402 (MEXU); Flores-Franco 4442 (MEXU); Téllez-Valdés 9989 (MEXU); Téllez-Valdés 10009 (MEXU); Téllez-Valdés 10085 (MEXU); Téllez-Valdés 10109 (MEXU); TéllezValdés 10112 (MEXU)].

Ageratina beamanii B.L. Turner [Breedlove 44463 (TEX)].

Ageratina blepharilepis (Sch. Bip.) R.M. King \& H. Rob. [Flores-Franco 1689 (MEXU); FloresFranco 2449 (MEXU); Ramírez-Rodríguez 798 (MEXU); Téllez-Valdés 11426 (MEXU)]. Ageratina calaminthifolia (Kunth) R.M. King \& H. Rob. [(Citado por Turner, 1997)].

Ageratina choricephala (B.L. Rob.) R.M. King \& H. Rob. [Téllez-Valdés 11483 (MEXU)]. Ageratina conspicua (Kunth \& Bouché) R.M. King \& H. Rob. [Magaña-Rueda 139 (MEXU); McVaugh 12130 (MEXU)].

Ageratina grashoffii B.L. Turner [(Citado por Turner, 1997)].

Ageratina jaliscensis (B.L. Rob.) Gage [(Citado por Turner, 1997)].

Ageratina lasioneura (Hook. \& Arn.) R.M. King \& H. Rob. [Calzada 19176 (MEXU); Flores-

Franco 2495 (MEXU); Gentry 10486 (MEXU); McVaugh 12125 (MEXU); Tenorio-Lezama 15588 (MEXU); Tenorio-Lezama 15648 (MEXU)].

Ageratina leptodictyon (A. Gray) R.M. King \& H. Rob. [McVaugh 16430 (MEXU); TenorioLezama 15970 (MEXU)].

Ageratina mairetiana (DC.) R.M. King \& H. Rob. var. mairetiana [Téllez-Valdés 10087 (MEXU)].

Ageratina malacolepis (B.L. Rob.) R.M. King \& H. Rob. [Calzada 19178 (EBCH, ENCB, MEXU, MICH, MO, US, XAL); Flores-Franco 1920 (MEXU); Magaña-Rueda 120 (MEXU);

Ramírez-Rodríguez 855 (MEXU)].

Ageratina muelleri (Sch. Bip. ex Klatt) R.M. King \& H. Rob. [(Citado por Turner, 1997)]. Ageratina palmeri (A. Gray) Gage [Citado por Turner, 1997)].

Ageratina paupercula (A. Gray) R.M. King \& H. Rob. [(Citado por Turner, 1997)].

Ageratina pazcuarensis (Kunth) R.M. King \& H. Rob. [(Citado por Turner, 1997)].

Ageratina pichinchensis (Kunth) R.M. King \& H. Rob. [Téllez-Valdés 10320 (MEXU)].

Ageratina triniona (McVaugh) R.M. King \& H. Rob. [Flores-Franco 4517 (MEXU); TenorioLezama 16503 (MEXU)].

Ageratum conyzoides L. [Graber 2 (Citado por McVaugh, 1984); McVaugh 556 (Citado por McVaugh, 1984); Palmer 1834 (Citado por McVaugh, 1984); Palmer 1850 (Citado por McVaugh, 1984); Téllez-Valdés 10256 (MEXU)].

Ageratum corymbosum Zuccagni ex Pers. [Aguilar-Orozco 340 (MEXU, UAN); BenítezParedes 3610 (MEXU); Benítez-Paredes 3955 (MEXU); Breckon 1004 (MEXU); Calzada 18839 (MEXU, MICH); Calzada 19078 (MEXU); Calzada 19186 (MEXU); Cronquist 9593 (MEXU); Flores-Franco 1314 (MEXU); Flores-Franco 1469 (MEXU); Flores-Franco 2905 (MEXU); Flores-Franco 3023 (ENCB, MEXU, MICH, MO, US, XAL); Flores-Franco 3161 (ENCB, MEXU, MICH, MO, US, XAL); Flores-Franco 3962 (MEXU); Flores-Franco 3987 
(MEXU); Flores-Franco 4030 (MEXU); Flores-Franco 4044 (MEXU); Flores-Franco 4323 (ENCB, MEXU, MICH, MO, US, XAL); Flores-Franco 4367 (MEXU); Flores-Franco 4409 (MEXU); Flores-Franco 4413 (MEXU), MICH; Flores-Franco 4452 (ENCB, MEXU, MICH, MO); La Duke 462 (MEXU); McVaugh 526 (MICH); Norris 13849 (MEXU); RzedowskiRotter 17843 (MEXU); Téllez-Valdés 9002 (MEXU); Téllez-Valdés 9288 (MEXU); TéllezValdés 9837 (MEXU); Téllez-Valdés 9847 (MEXU); Téllez-Valdés 9929 (MEXU); TéllezValdés 10822 (MEXU); Téllez-Valdés 10933 (MEXU); Téllez-Valdés 12034 (MEXU); Téllez-Valdés 12083 (MEXU); Téllez-Valdés 12533 (MEXU); Téllez-Valdés 12877 (ENCB, MEXU, MICH, MO); Téllez-Valdés 13031 (ENCB, MEXU, MICH); TenorioLezama 16055 (MEXU); Tenorio-Lezama 16350 (MEXU); Tenorio-Lezama 16534 (MEXU); Tenorio-Lezama 16995 (MEXU)].

Ageratum houstonianum Mill. [Ramírez-Rodríguez 835 (MEXU); Téllez-Valdés 11862 (MEXU)].

Aldama dentata Llave var. dentata [Flores-Franco 3475 (MEXU, UAN); King 3668 (MEXU); King 3681 (MEXU); Stuessy 3055 (MEXU)].

Alloispermum michoacanum (B.L. Rob.) B.L. Turner var. liebmannii (Sch. Bip. ex Klatt) B.L. Turner [Flores-Franco 3240 (MEXU, MICH); Téllez-Valdés 11612 (MEXU); Téllez-Valdés 11615 (MEXU)].

Alloispermum palmeri (S. Watson ex A. Gray) Fernández \& Urbatsch var. lancifolium (Urbatsch \& B.L. Turner) Fernández \& Urbatsch [Norris 14259 (Citado por McVaugh, 1984); Rose 3413 (Citado por McVaugh, 1984)].

Alloispermum palmeri (S. Watson ex A. Gray) Fernández \& Urbatsch var. palmeri [FloresFranco 2086 (MEXU); Téllez-Valdés 10747 (MEXU)].

Alloispermum scabrifolium (Hook. \& Arn.) H. Rob. [Calzada 19206 (BM, EBCH, ENCB, F, FCME, GH, IBUG, K, MEXU, MICH, MO, US, XAL); Flores-Franco 2490 (MEXU); FloresFranco 3508 (MEXU, UAN); Flores-Franco 4520 (MEXU); Flores-Franco 4534 (MEXU); González-Flores 550 (MEXU); Téllez-Valdés 10100 (MEXU); Téllez-Valdés 10317 (MEXU); Téllez-Valdés 11680 (MEXU); Tenorio-Lezama 15587 (MEXU); TenorioLezama 15616 (MEXU)].

Alloispermum scabrum (Lag.) H. Rob. [Flores-Franco 1460 (MEXU); Téllez-Valdés 9748 (MEXU); Téllez-Valdés 9931 (MEXU); Téllez-Valdés 11557 (MEXU); Téllez-Valdés 11564 (MEXU); Téllez-Valdés 11584 (MEXU); Téllez-Valdés 13033 (MEXU); TenorioLezama 16145 (MEXU); Tenorio-Lezama 16526 (MEXU)].

Ambrosia maritima L. [Chiang-Cabrera 982 (MEXU)].

Archibaccharis hirtella (DC.) Heering [Téllez-Valdés 9775 (MEXU)].

Archibaccharis schiedeana (Benth.) J.D. Jacks. [Calzada 18907 (ENCB, MEXU, MICH, MO); Calzada 18937 (MEXU); Flores-Franco 3969 (MEXU); Flores-Franco 4112 (MEXU); Flores-Franco 4325 (ENCB, MEXU, MICH, MO, US, XAL); Flores-Franco 4403 (MEXU); Flores-Franco 4456 (MEXU); McVaugh 13339 (MEXU)].

Artemisia ludoviciana Nutt. [Aguilar-Orozco 329 (MEXU, UAN); Aguilar-Orozco 422 (MEXU, UAN)]. 
Aster subulatus Michx. var. subulatus [Flores-Franco 1139 (MEXU); Novelo-Retana 618 (MEXU); Novelo-Retana 662 (MEXU); Novelo-Retana 672 (MEXU); Téllez-Valdés 11229 (MEXU); Téllez-Valdés 12880 (MEXU)].

Astranthium xylopodum Larsen [Calzada 18773 (MEXU, MICH); McVaugh 14922 (MEXU); McVaugh 15303 (MEXU); Webster 17108 (MEXU)].

Axiniphyllum durangense B.L. Turner [Flores-Franco 1410 (MEXU); Téllez-Valdés 12251 (MEXU); Tenorio-Lezama 16466 (MEXU)].

Baccharis heterophylla Kunth [Breedlove 45437 (MEXU); Téllez-Valdés 11616 (MEXU); Téllez-Valdés 12244 (MEXU)].

Baccharis occidentalis S.F. Blake [Gentry 10794 (MEXU); McVaugh 14923 (MEXU)].

Baccharis pteronioides DC. [Téllez-Valdés 10415 (MEXU)].

Baccharis salicifolia (Ruiz \& Pav.) Pers. [Breedlove 45465 (MEXU); Flores-Franco 1429 (MEXU); Flores-Franco 4457 (MEXU); Norris 13905 (MEXU); Téllez-Valdés 10566 (MEXU); Téllez-Valdés 12511 (MEXU); Tenorio-Lezama 15965 (MEXU); TenorioLezama 16496 (MEXU); Webster 15759 (MEXU)].

Baccharis squarrosa Kunth [McVaugh 25752 (MEXU, MICH)].

Baccharis thesioides Kunth [Flores-Franco 1161 (MEXU); Tenorio-Lezama 17011 (MEXU)]. Baccharis trinervis (Lam.) Pers. [Flores-Franco 3313 (MEXU); Flores-Franco 3328 (MEXU); Flores-Franco 3353 (MEXU); Flores-Franco 3398 (MEXU, MICH); Norris 13292 (ENCB, $\mathrm{MICH})$.

Bidens alba (L.) DC. var. alba [Flores-Franco 1370 (MEXU)].

Bidens aurea (Aiton) Sherff [Flores-Franco 1349 (MEXU); Téllez-Valdés 12523 (MEXU); Tenorio-Lezama 16406 (MEXU)].

Bidens cordylocarpa (A. Gray) D.J. Crawford [Tenorio-Lezama 16110 (MEXU)].

Bidens ferulifolia (Jacq.) DC. [Tenorio-Lezama 15959 (MEXU); Tenorio-Lezama 16025 (MEXU)].

Bidens laevis (L.) Britton, Stearn \& Pogg. [Téllez-Valdés 11240 (MEXU)].

Bidens odorata Cav. var. odorata [Aguilar-Orozco 322 (MEXU, UAN); Aguilar-Orozco 346

(MEXU, UAN); Aguilar-Orozco 398 (MEXU, UAN); Aguilar-Orozco 428 (MEXU, UAN); Aguilar-Orozco 499 (MEXU, UAN); Flores-Franco 4127 (MEXU); Flores-Franco 4255 (MEXU); Flores-Franco 4399 (MEXU); Téllez-Valdés 9580 (MEXU); Téllez-Valdés 10945 (MEXU); Téllez-Valdés 11453 (MEXU); Téllez-Valdés 11488 (MEXU); Téllez-Valdés 11518 (MEXU); Téllez-Valdés 11552 (MEXU); Téllez-Valdés 11613 (MEXU); TenorioLezama 16337 (MEXU); Tenorio-Lezama 16425 (MEXU)].

Bidens odorata Cav. var. rosea (Sch. Bip.) Melchert [Flores-Franco 1338 (MEXU)].

Bidens pilosa L. var. pilosa [Bravo-Bolaños 2252 (MEXU); Novelo-Retana 881 (MEXU); Téllez-Valdés 9361 (MEXU); Téllez-Valdés 9486 (MEXU); Téllez-Valdés 10118 (MEXU)].

Bidens reptans (L.) G. Don ex Sweet var. urbanii (Greenm.) O.E. Schulz [Chiang-Cabrera 1117 (MEXU)].

Bidens riparia Kunth var. refracta (Brandegee) O.E. Schulz [Flores-Franco 1073 (MEXU)]. Bidens rostrata Melchert [Flores-Franco 1354 (MEXU); Flores-Franco 1369 (MEXU); FloresFranco 1599 (MEXU); Tenorio-Lezama 16007 (MEXU); Tenorio-Lezama 16385 (MEXU); Tenorio-Lezama 16704 (MEXU)]. 
Bidens serrulata (Poir.) Desf. var. serrulata [Téllez-Valdés 11515 (MEXU); Téllez-Valdés 11558 (MEXU)].

Bidens triplinervia Kunth var. macrantha (Wedd.) Sherff [Santana-Michel 2386 (IBUG, MEXU)].

Bolanosa coulteri A. Gray [Calzada 18848 (MEXU); Flores-Franco 1707 (MEXU); Schubert 2066 (MEXU); Téllez-Valdés 9787 (MEXU); Téllez-Valdés 10792 (MEXU); Téllez-Valdés 11083 (MEXU); Téllez-Valdés 12443 (MEXU); Tenorio-Lezama 16635 (MEXU); Webster 15721 (MEXU)].

Brickellia cardiophylla B.L. Rob. [(Citado por Turner, 1997)].

Brickellia coulteri A. Gray var. adenopoda (B.L. Rob.) B.L. Turner [Flores-Franco 1782 (MEXU); Téllez-Valdés 11362 (MEXU)].

Brickellia cuspidata A. Gray [Téllez-Valdés 9590 (MEXU); Tenorio-Lezama 16714 (MEXU); Tenorio-Lezama 16897 (MEXU)].

Brickellia diffusa (Vahl) A. Gray [Téllez-Valdés 9864 (MEXU)].

Brickellia filipes B.L. Rob. [Téllez-Valdés 11722 (MEXU)].

Brickellia jaliscensis McVaugh [Flores-Franco 2469 (MEXU)].

Brickellia lanata (DC.) A. Gray [Calzada 19113 (MEXU); Calzada 19144 (MEXU); FloresFranco 1608 (MEXU); Flores-Franco 1834 (MEXU); Flores-Franco 3351 (MEXU); Paray s.n. (MEXU)].

Brickellia magnifica McVaugh [Téllez-Valdés 10161 (MEXU)].

Brickellia oliganthes (Less.) A. Gray [Benítez-Paredes 3709 (MEXU)].

Brickellia paniculata (Mill.) B.L. Rob. [Calzada 19225 (EBCH, ENCB, FCME, MEXU, MICH, MO, US, XAL); Flores-Franco 3283 (MEXU); Rose 14 (US), Rose 337 (US); Téllez-Valdés 9991 (MEXU); Téllez-Valdés 11238 (MEXU, RSA)].

Brickellia pringlei A. Gray [Palmer s.n. (US)].

Brickellia scoparia (DC.) A. Gray var. scoparia [McVaugh 25758 (Citado por McVaugh, 1984)].

Brickellia secundiflora (Lag.) A. Gray var. nepetifolia (Kunth) B.L. Rob. [Téllez-Valdés 11354 (MEXU)].

Brickellia seemannii A. Gray [(Citado por Turner, 1997)].

Brickellia subuligera (Schauer) B.L. Turner [Flores-Franco 1695 (MEXU)].

Brickellia worthingtonii B.L. Turner [(Citado por Turner, 1997)].

Calea jamaicensis (L.) L. [Téllez-Valdés 9565 (MEXU)].

Calea ternifolia Kunth var. ternifolia [Téllez-Valdés 9281 (MEXU); Téllez-Valdés 10821 (MEXU)].

Calea urticifolia (Mill.) DC. var. urticifolia [Benítez-Paredes 3636 (MEXU); Calzada 19204 (ENCB, MEXU, MICH, MO, XAL); Flores-Franco 941 (MEXU); Flores-Franco 1685 (MEXU); Flores-Franco 1919 (MEXU); Flores-Franco 2513 (EBCH, ENCB, MEXU, MICH, MO, US, XAL); Flores-Franco 3362 (MEXU); Flores-Franco 3519 (MEXU); Flores-Franco 3734 (MEXU); Flores-Franco 4223 (MEXU); García-Pérez 917 (MEXU); Gentry 19520 (MEXU); McVaugh 12073 (MEXU); Téllez-Valdés 4149 (MEXU); Téllez-Valdés 10262 (MEXU); Téllez-Valdés 10265 (MEXU); Téllez-Valdés 10411 (MEXU); Téllez-Valdés 12529 (MEXU); Tenorio-Lezama 15599 (MEXU)]. 
Calendula officinalis L. [Ruenes 78 (MEXU)].

Carminatia recondita McVaugh [Flores-Franco 1464 (MEXU); Flores-Franco 4028 (ENCB, MEXU, MICH); Téllez-Valdés 9897 (MEXU); Téllez-Valdés 11059 (MEXU); TenorioLezama 16530 (MEXU)].

Carminatia tenuiflora DC. [Campos-Villanueva 5027 (MEXU, XAL); Flores-Franco 1586 (MEXU); Flores-Franco 1646 (MEXU)].

Centaurea rothrockii Greenm. [Breedlove 44582 (MEXU)].

Chaptalia runcinata Kunth [Rose 2022 (Citado por McVaugh, 1984)].

Chaptalia texana Greene [(Citado por Nesom, 1995)].

Chloracantha spinosa (Benth.) G.L. Nesom var. jaliscensis (McVaugh) Sundberg [BenítezParedes 3792 (MEXU); Flores-Franco 963 (MEXU); McVaugh 19049 (MICH); TenorioLezama 16618 (MEXU)].

Chrysanthellum indicum DC. var. mexicanum (Greenm.) B.L. Turner [Téllez-Valdés 8948 (MEXU)].

Cirsium anartiolepis Petrak [Flores-Franco 3272 (MEXU); Téllez-Valdés 10120 (MEXU); Téllez-Valdés 12602 (EBCH, ENCB, FCME, MEXU, MICH, MO, US, XAL)].

Cirsium ehrenbergii Sch. Bip. [Téllez-Valdés 11573 (MEXU)].

Cirsium grahamii A. Gray [Diguet s.n. (Citado por McVaugh, 1984); Rose 2152 (US)].

Cirsium rhaphilepis (Hemsl.) Petrak [Téllez-Valdés 10510 (MEXU)].

Cirsium velatum (S. Watson) Petrak [McVaugh 19408 (Citado por McVaugh, 1984)].

Clibadium arboreum Donn. Sm. [Flores-Franco 2909 (MEXU); Flores-Franco 4299 (MEXU); Flores-Franco 4311 (MEXU); Téllez-Valdés 12035 (MEXU)].

Conyza bonariensis (L.) Cronquist [Téllez-Valdés 9769 (MEXU)].

Conyza canadensis (L.) Cronquist var. glabrata (A. Gray) Cronquist [Benítez-Paredes 3771

(MEXU); Flores-Franco 3497 (MEXU, UAN); Flores-Franco 3782 (MEXU); King 3685

(MEXU); Téllez-Valdés 9382 (MEXU); Téllez-Valdés 10151 (MEXU)].

Conyza canadensis (L.) Cronquist var. pusilla (Nutt.) Cronquist [Feddema 614 (Citado por McVaugh, 1984); King 3685 (Citado por McVaugh, 1984); Palmer s.n. (Citado por McVaugh, 1984)].

Conyza coronopifolia Kunth [Rose 2026 (Citado por McVaugh, 1984)].

Coreopsis cuneifolia Greenm. [Tenorio-Lezama 16697 (MEXU)].

Coreopsis petrophila A. Gray [Tenorio-Lezama 17013 (MEXU)].

Coreopsis rudis (Benth.) Hemsl. [Flores-Franco 2374 (MEXU); Ramírez-Rodríguez 718 (MEXU); Téllez-Valdés 11617 (MEXU)].

Cosmos bipinnatus Cav. [Breedlove 44563 (MEXU); Calzada 18774 (MEXU, MICH); FloresFranco 1353 (MEXU); Flores-Franco 3095 (MEXU, MICH); Flores-Franco 3237 (MEXU, $\mathrm{MICH}$ ); Téllez-Valdés 9278 (MEXU); Téllez-Valdés 11586 (MEXU)]. 
Cosmos carvifolius Benth. [Flores-Franco 2899 (EBCH, ENCB, FCME, MEXU, MICH, MO, US, XAL); Flores-Franco 3207 (ENCB, MEXU, MICH); Flores-Franco 3295 (MEXU); Flores-Franco 3907 (MEXU); Flores-Franco 3994 (MEXU); Flores-Franco 4386 (MEXU, $\mathrm{MICH}$ ); Flores-Franco 4419 (MEXU); Flores-Franco 4454 (ENCB, MEXU, MICH, MO); Flores-Franco 4468 (MEXU); González-Flores 1324 (MEXU); Téllez-Valdés 9746 (MEXU); Téllez-Valdés 10101 (MEXU); Téllez-Valdés 12379 (ENCB, MEXU, MICH, MO, US, XAL); Tenorio-Lezama 16411 (MEXU)].

Cosmos crithmifolius Kunth [Flores-Franco 1292 (MEXU); Flores-Franco 3237 (MEXU, MICH); Flores-Franco 3295 (MEXU); Flores-Franco 4539 (MEXU); González-Flores 1324 (MEXU); McVaugh 16432 (MEXU); Rose 2154 (MEXU)].

Cosmos diversifolius Otto ex Knowles \& Westc. var. diversifolius [Tenorio-Lezama 16410 (MEXU)].

Cosmos intercedens Sherff [Téllez-Valdés 9577 (MEXU); Téllez-Valdés 9585 (MEXU)].

Cosmos linearifolius (Sch. Bip.) Hemsl. var. linearifolius [Rose 2154 (Citado por McVaugh, 1984)].

Cosmos montanus Sherff [Rose 3433 (Citado por McVaugh, 1984)].

Cosmos parviflorus (Jacq.) Pers. [Flores-Franco 1354 (MEXU); Téllez-Valdés 9134 (MEXU); Téllez-Valdés 11570 (MEXU); Tenorio-Lezama 16424 (MEXU)].

Cosmos pringlei B.L. Rob. \& Fernald [Rose 2120 (Citado por McVaugh, 1984)].

Cosmos scabiosoides Kunth var. scabiosoides [Tenorio-Lezama 15960 (MEXU)].

Cosmos sulphureus Cav. [Benítez-Paredes 3718 (MEXU); Bravo-Bolaños 2082 (MEXU); Bravo-Hollis 9238 (MEXU); Calzada 18875 (ENCB, MEXU, MICH); Calzada 18964 (MEXU, MICH); García-Pérez 911 (MEXU); Kimnach 2288 (MEXU); Téllez-Valdés 9494 (MEXU); Téllez-Valdés 9800 (MEXU); Téllez-Valdés 10836 (MEXU); Téllez-Valdés 12405 (ENCB, MEXU, MICH, MO); Téllez-Valdés 12528 (MEXU); Tenorio-Lezama 16086 (MEXU); Villaseñor-Ríos 714 (MEXU)].

Dahlia coccinea Cav. [Aguilar-Orozco 282 (ENCB, MEXU, MICH, MO, UAN, XAL); FloresFranco 965 (MEXU, RSA); Flores-Franco 1307 (MEXU); Flores-Franco 2628 (MEXU); Flores-Franco 2833 (MEXU, MICH); Flores-Franco 3715 (ENCB, MEXU, MICH); FloresFranco 3738 (ENCB, MEXU, MICH); Flores-Franco 3881 (ENCB, MEXU, MICH, MO, US, XAL); Flores-Franco 3978 (MEXU); Flores-Franco 4056 (ENCB, MEXU, MICH, MO); Flores-Franco 4122 (ENCB, MEXU, MICH); Flores-Franco 4209 (MEXU, MICH); FloresFranco 4349 (ENCB, MEXU, MICH); Téllez-Valdés 9224 (MEXU); Téllez-Valdés 10947 (MEXU); Téllez-Valdés 12270 (MEXU)].

Decachaeta haenkeana DC. [Ferris 5716 (MEXU); Téllez-Valdés 11036 (MEXU); TéllezValdés 11089 (MEXU)].

Decachaeta ovatifolia (DC.) R.M. King \& H. Rob. [Téllez-Valdés 9418 (MEXU); Téllez-Valdés 11425 (MEXU)].

Decachaeta scabrella (B.L. Rob.) R.M. King \& H. Rob. [Flores-Franco 1459 (MEXU); Ramírez-Rodríguez 776 (ENCB, MEXU, MICH, MO, XAL); Téllez-Valdés 12470 (MEXU)].

Delilia biflora (L.) Kuntze [Benítez-Paredes 2953 (MEXU); Ramírez-Rodríguez 763 (MEXU); Téllez-Valdés 9888 (MEXU); Téllez-Valdés 10195 (MEXU)]. 
Desmanthodium fruticosum Greenm. [Breedlove 44351 (MEXU); Flores-Franco 3927 (MEXU); Flores-Franco 4222 (MEXU); McVaugh 13360 (MEXU)].

Dyssodia tagetiflora Lag. [Téllez-Valdés 9581 (MEXU); Téllez-Valdés 10998 (MEXU)].

Eclipta prostrata (L.) L. [Benítez-Paredes 3371 (MEXU); Norris 13861 (MEXU); NoveloRetana 658 (MEXU); Ramírez-Rodríguez 838 (MEXU); Rose 1424 (MEXU); TéllezValdés 12539 (MEXU); Téllez-Valdés 12894 (MEXU)].

Egletes viscosa (L.) Less. var. viscosa [Téllez-Valdés 10345 (MEXU)].

Elephantopus mollis Kunth [Aguilar-Orozco 476 (MEXU, UAN); Benítez-Paredes 3691 (MEXU); Calzada 18884 (MEXU); Ferris 5925 (CAS, MEXU); Flores-Franco 4292 (ENCB, MEXU, MICH, MO); Téllez-Valdés 9422 (MEXU)].

Encelia angustifolia Greenm. [Rose 3453 (US, GH)].

Erechtites hieraciifolia (L.) Raf. ex DC. var. cacalioides (Fisch. ex Spreng.) Griseb. [TéllezValdés 9520 (MEXU); Téllez-Valdés 11247 (MEXU)].

Erigeron delphinifolius Willd. [Téllez-Valdés 11561 (MEXU); Téllez-Valdés 11571 (MEXU)]. Erigeron exilis A. Gray [Diguet s.n. (Citado por McVaugh, 1984); McVaugh 23535 (Citado por McVaugh, 1984)].

Erigeron griseus (Greenm.) G.L. Nesom [Flores-Franco 2217 (MEXU); Flores-Franco 2260 (MEXU)].

Erigeron karvinskianus DC. [Flores-Franco 1885 (MEXU); Flores-Franco 2087 (MEXU); Flores-Franco 2366 (MEXU)].

Erigeron longipes DC. [Calzada 18769 (MEXU); Flores-Franco 2835 (ENCB, MEXU, MICH); Soule 2711 (MEXU, TEX)].

Erigeron polycephalus (Larsen) G.L. Nesom [Téllez-Valdés 9388 (MEXU); Téllez-Valdés 11899 (MEXU)].

Erigeron velutipes Hook. \& Arn. [Téllez-Valdés 10001 (MEXU); Téllez-Valdés 10109 (MEXU); Téllez-Valdés 10375 (MEXU)].

Eryngiophyllum pinnatisectum P.G. Wilson [Ron s.n. (IBUG)].

Eupatoriastrum triangulare (DC.) B.L. Rob. [Stuessy 4120 (MEXU); Téllez-Valdés 10944 (MEXU)].

Eupatorium albicaule Sch. Bip. ex Klatt [Maltby 40 (Citado por McVaugh, 1984)].

Eupatorium collinum DC. [Benítez-Paredes 3033 (MEXU); Benítez-Paredes 3668 (MEXU); Chiang-Cabrera 1199 (MEXU); Flores-Franco 1612 (MEXU); Flores-Franco 2477 (MEXU); Flores-Franco 3150 (MEXU, MICH); Téllez-Valdés 11365 (MEXU); TenorioLezama 16668 (MEXU)].

Eupatorium glaberrimum DC. [Téllez-Valdés 9427 (MEXU)].

Eupatorium haenkeanum DC. [Martínez-Esquivel 711 (MEXU)].

Eupatorium hebebotryum (DC.) Hemsl. [Tenorio-Lezama 16612 (MEXU)].

Eupatorium monanthum Sch. Bip. [Ayers 105 (MEXU); Flores-Franco 2029 (MEXU); TéllezValdés 4151 (MEXU); Téllez-Valdés 10289 (MEXU); Téllez-Valdés 11314 (MEXU);

Téllez-Valdés 11810 (MEXU)]. 
Eupatorium nelsonii B.L. Rob. [Flores-Franco 1436 (MEXU); Téllez-Valdés 9739 (MEXU)]. Eupatorium odoratum L. [Benítez-Paredes 2963 (MEXU); Calzada 19075 (MEXU); TéllezValdés 9378 (MEXU); Téllez-Valdés 11246 (MEXU); Téllez-Valdés 11322 (MEXU); Téllez-Valdés 11643 (MEXU); Téllez-Valdés 11745 (MEXU)].

Eupatorium ovaliflorum Hook. \& Arn. [Benítez-Paredes 3021 (MEXU); Calzada 19098 (MEXU); Calzada 19120 (MEXU); Croat 45232 (MEXU); González-Flores 673 (MEXU, UAN); González-Flores 896 (MEXU, UAN); Téllez-Valdés 9459 (MEXU); Téllez-Valdés 10266 (MEXU); Téllez-Valdés 11127 (ENCB, MEXU, MICH); Téllez-Valdés 12574 (MEXU, MICH); Croat 45232 (MEXU)].

Eupatorium pulchellum Kunth [Flores-Franco 1351 (MEXU); Flores-Franco 1352 (MEXU); Flores-Franco 1366 (MEXU); Tenorio-Lezama 16409 (MEXU)].

Eupatorium pycnocephalum Less. [Flores-Franco 898 (MEXU); Flores-Franco 1918 (MEXU); Flores-Franco 2018 (MEXU); Téllez-Valdés 10147 (MEXU); Tenorio-Lezama 15617 (MEXU)].

Eupatorium quadrangulare DC. [Flores-Franco 3383 (MEXU); Flores-Franco 4166 (MEXU, MICH); Téllez-Valdés 4141 (MEXU); Téllez-Valdés 9367 (MEXU); Téllez-Valdés 11212 (MEXU); Téllez-Valdés 11635 (MEXU); Tenorio-Lezama 16625 (MEXU)].

Eupatorium sagittatum A. Gray [Clarke 681231-16 (Citado por McVaugh, 1984)].

Eupatorium solidaginifolium A. Gray [Flores-Franco 1613 (MEXU); Flores-Franco 1614 (MEXU); Téllez-Valdés 12439 (MEXU)].

Eupatorium trinervium Sch. Bip. [McVaugh 784 (Citado por McVaugh, 1984)].

Galeana pratensis (Kunth) Rydb. [Flores-Franco 968 (MEXU); Ramírez-Rodríguez 502 (MEXU); Ramírez-Rodríguez 1107 (MEXU); Téllez-Valdés 9349 (MEXU); Téllez-Valdés 12423 (MEXU)].

Galinsoga parviflora Cav. [Flores-Franco 1619 (MEXU); Flores-Franco 2372 (MEXU); FloresFranco 4020 (MEXU); Flores-Franco 4046 (MEXU); Flores-Franco 4110 (MEXU); FloresFranco 4397 (MEXU); Flores-Franco 4473 (MEXU); Stuessy 3060 (MEXU); Téllez-Valdés 11473 (MEXU); Téllez-Valdés 11806 (MEXU)].

Galinsoga quadriradiata Ruiz \& Pav. [Bravo-Hollis 9246 (MEXU); Flores-Franco 4400 (MEXU); Flores-Franco 4509 (MEXU)].

Gamochaeta americana (Mill.) Cabrera [Flores-Franco 2861 (MEXU, MICH)].

Gnaphalium indicum L. [Palmer s.n. (US)].

Gnaphalium pacificum G.L. Nesom [Flores-Franco 1716 (MEXU)].

Guardiola carinata B. L. Rob. [Magaña-Rueda 119 (MEXU); Téllez-Valdés 9173 (MEXU); Téllez-Valdés 10687 (MEXU); Téllez-Valdés 12069 (MEXU)].

Guardiola mexicana Humb. \& Bonpl. var. angustifolia (A. Gray) McVaugh [Breedlove 61525 (MEXU); Flores-Franco 1160 (MEXU); Flores-Franco 1336 (MEXU); Téllez-Valdés 11501 (MEXU); Téllez-Valdés 12164 (MEXU); Téllez-Valdés 12243 (MEXU); Tenorio-Lezama 16058 (MEXU); Tenorio-Lezama 16335 (MEXU)].

Guardiola mexicana Humb. \& Bonpl. var. mexicana [Daniel 4780; Téllez-Valdés 9312 (MEXU); Téllez-Valdés 10039 (MEXU); Téllez-Valdés 10082; Téllez-Valdés 11337 (MEXU)]. 
Guardiola rosei B.L. Rob. [Arcadia-Alvarez 199 (MEXU); Breedlove 45237 (MEXU)]. Guardiola tulocarpus A. Gray [Calzada 18777 (ENCB, MEXU, MICH, US); Calzada 18859 (ENCB, MEXU, MICH); Calzada 19112 (MEXU); Flores-Franco 934 (MEXU); FloresFranco 2502 (MEXU, MICH); Flores-Franco 3222 (ENCB, MEXU, MICH, MO, XAL); Flores-Franco 3282 (MEXU); Paray s.n. (MEXU); Rzedowski-Rotter 17877 (MEXU); Téllez-Valdés 8233 (MEXU); Téllez-Valdés 9566 (MEXU); Téllez-Valdés 10729 (MEXU); Téllez-Valdés 11697 (MEXU, MICH); Téllez-Valdés 12590 (EBCH, ENCB, MEXU, MICH, MO, US, XAL); Téllez-Valdés 12598 (ENCB, MEXU, MICH); Téllez-Valdés 12910 (MEXU); Tenorio-Lezama 15688 (MEXU); Tenorio-Lezama 16888 (MEXU); Webster 19880 (MEXU)].

Gutierrezia sericocarpa (A. Gray) M.A. Lane [Breedlove 44601 (MEXU)].

Gymnocoronis latifolia Hook. \& Arn. [Beechey s.n. (K)].

Helenium thurberi A. Gray [Citado por Bierner, 1972].

Helianthus annuus L. [Ruenes 70 (MEXU); Solís 649 (MEXU)].

Heliomeris longifolia (B.L. Rob. \& Greenm.) Cockerell var. longifolia [Téllez-Valdés 11380 (MEXU); Téllez-Valdés 11476 (MEXU)].

Heliopsis novogaliciana B.L. Turner [Gentry 10775 (MEXU)].

Heliopsis parviceps S.F. Blake [Flores-Franco 1628 (MEXU)].

Heliopsis procumbens Hemsl. [McVaugh 15189 (MEXU); Webster 17136 (MEXU)].

Heterotheca inuloides Cass. var. rosei B. Wagenk. [Calzada 19174 (MEXU); Flores-Franco 1886 (MEXU); Flores-Franco 1928 (MEXU); Flores-Franco 2828 (ENCB, MEXU, MICH); Flores-Franco 3481 (MEXU, UAN); Flores-Franco 3502 (MEXU, UAN); González-Flores 706 (MEXU, UAN); Téllez-Valdés 10043 (MEXU); Téllez-Valdés 10376 (MEXU); TéllezValdés 10559 (MEXU); Tenorio-Lezama 15656 (MEXU); Tenorio-Lezama 17016 (MEXU)].

Hieracium abscissum Less. [Rose s.n. (Citado por McVaugh, 1984)].

Hieracium crepidispermum Fries [Breedlove 45080 (MEXU)].

Hieracium fendleri Sch. Bip. subsp. ostreophyllum (Standl. \& Steyerm.) Beaman [TéllezValdés 11025 (MEXU)].

Hieracium pringlei A. Gray [Rose 2044 (Citado por McVaugh, 1984)].

Hofmeisteria dissecta (Hook. \& Arn.) R.M. King \& H. Rob. [Citado por Turner, 1997)].

Hofmeisteria mexiae (B.L. Rob.) B.L. Turner [McVaugh 817 (Citado por McVaugh, 1984); McVaugh 15179 (Citado por McVaugh, 1984)].

Hofmeisteria urenifolia (Hook. \& Arn.) Walp. [Flores-Franco 953 (MEXU); Téllez-Valdés 11805 (MEXU)].

lostephane heterophylla (Cav.) Hemsl. [Flores-Franco 1400 (MEXU); Téllez-Valdés 10754 (MEXU); Tenorio-Lezama 16455 (MEXU)].

lostephane madrensis (S. Watson) Strother [Breedlove 61454 (MEXU)]. 
Jaegeria hirta (Lag.) Less. [Breedlove 44473 (MEXU); Flores-Franco 1372 (MEXU); FloresFranco 1379 (MEXU); Flores-Franco 4113 (MEXU); Téllez-Valdés 10470 (MEXU); TéllezValdés 11592 (MEXU)].

Jaegeria macrocephala Less. [Flores-Franco 2256 (MEXU); King 3670 (MEXU); Palacios s.n. (MEXU)].

Jaegeria pedunculata Hook. \& Arn. [Tenorio-Lezama 16427 (ENCB, MEXU, MICH, MO)].

Jaumea peduncularis (Hook. \& Arn.) Oliver \& Hiern. ex Oliver [Benítez-Paredes 2959 (MEXU); Benítez-Paredes 2987 (MEXU); Benítez-Paredes 3793 (MEXU); Calzada 18862 (MEXU); Calzada 19100 (MEXU); Flores-Franco 1961 (MEXU); Téllez-Valdés 11798 (MEXU); Téllez-Valdés 12918 (MEXU)].

Lactuca intybacea Jacq. [Martínez-Esquivel 1016 (MEXU)].

Laennecia confusa (Cronquist) G.L. Nesom [Téllez-Valdés 11517 (MEXU)].

Laennecia gnaphalioides (Kunth) Cass. [Tenorio-Lezama 16695 (MEXU)].

Laennecia sophiifolia (Kunth) G.L. Nesom [Flores-Franco 2143 (MEXU); Ramírez-Rodríguez 572 (MEXU); Téllez-Valdés 11469 (MEXU)].

Lagascea angustifolia DC. [Benítez-Paredes 3705 (MEXU)].

Lagascea helianthifolia Kunth var. helianthifolia [Croat 45198 (MEXU, MO); Mayfield 1653

(MEXU, TEX); Téllez-Valdés 9365 (MEXU); Téllez-Valdés 9608 (MEXU); Téllez-Valdés

10063 (MEXU); Téllez-Valdés 11677 (MEXU); Tenorio-Lezama 15666 (MEXU)].

Lagascea helianthifolia Kunth var. levior (B.L. Rob.) B.L. Rob. [Calzada 19106 (MEXU);

Flores-Franco 2465 (MEXU); Flores-Franco 2494 (MEXU)].

Lagascea palmeri (B.L. Rob.) B.L. Rob. [Benítez-Paredes 3726 (MEXU)].

Lasianthaea ceanothifolia (Willd.) K.M. Becker var. ceanothifolia [Benítez-Paredes 3887

(MEXU); Benítez-Paredes 3934 (MEXU); Flores-Franco 1301 (MEXU); Flores-Franco 1618 (MEXU); Flores-Franco 4114 (MEXU); González-Flores 755 (MEXU, UAN); TéllezValdés 11497 (MEXU); Téllez-Valdés 12324 (MEXU); Téllez-Valdés 12527 (MEXU); Tenorio-Lezama 16057 (MEXU); Tenorio-Lezama 16395 (MEXU)].

Lasianthaea ceanothifolia (Willd.) K.M. Becker var. gracilis (W.W. Jones) K.M. Becker [Flores-Franco 969 (MEXU); Ramírez-Rodríguez 648 (MEXU); Téllez-Valdés 11094 (MEXU); Téllez-Valdés 11632 (MEXU); Téllez-Valdés 11964 (MEXU)].

Lasianthaea ceanothifolia (Willd.) K.M. Becker var. gradata (S.F. Blake) K.M. Becker [Feddema 950 (Citado por McVaugh, 1984)].

Lasianthaea ceanothifolia (Willd.) K.M. Becker var. verbenifolia (DC.) K.M. Becker [Becker 6 (MEXU, NY); Flores-Franco 2790 (MEXU, MICH); Salinas-Tovar 5131 (MEXU); TéllezValdés 11262 (MEXU)].

Lasianthaea fruticosa (L.) K.M. Becker var. fasciculata (DC.) K.M. Becker [Calzada 18871 (EBCH, ENCB, FCME, MEXU, MICH, MO, US, XAL); Flores-Franco 1468 (MEXU); Flores-Franco 3163 (ENCB, MEXU, MICH, MO); Flores-Franco 3923 (MEXU); FloresFranco 4092 (MEXU); Flores-Franco 4301 (MEXU); Flores-Franco 4327 (ENCB, MEXU, $\mathrm{MICH}, \mathrm{MO}, \mathrm{XAL}$ ); Flores-Franco 4467 (MEXU)].

Lasianthaea helianthoides DC. var. nayaritense K.M. Becker [García-Pérez 918 (MEXU); Téllez-Valdés 10767 (MEXU); Téllez-Valdés 12162 (MEXU)]. 
Lasianthaea macrocephala (Hook. \& Arn.) K.M. Becker [Benítez-Paredes 3011 (MEXU); Benítez-Paredes 3693 (MEXU); Benítez-Paredes 3963 (MEXU); Calzada 18900 (ENCB, MEXU, MICH, MO, XAL); Ferris 5787 (MEXU); Flores-Franco 985 (ENCB, MEXU, MICH, MO, XAL); Flores-Franco 1015 (MEXU); Flores-Franco 1284 (MEXU); Flores-Franco 1392 (MEXU); Flores-Franco 1525 (MEXU); Flores-Franco 3706 (MEXU); Flores-Franco 4085 (ENCB, MEXU, MICH, MO, US, XAL); Flores-Franco 4108 (MEXU); Flores-Franco 4174 (ENCB, MEXU, MICH); García-Pérez 915 (MEXU); King 3689 (MEXU); La Duke 391 (MEXU); McVaugh 13353 (MEXU); Paray 3393 (MEXU); Stuessy 3057 (MEXU); Stuessy 4113 (MEXU); Téllez-Valdés 9206 (MEXU); Téllez-Valdés 9296 (MEXU); TéllezValdés 9476 (MEXU); Téllez-Valdés 9813 (MEXU); Téllez-Valdés 10949 (MEXU); TéllezValdés 11249 (MEXU); Téllez-Valdés 11363 (MEXU); Téllez-Valdés 11440 (MEXU); Téllez-Valdés 12909 (MEXU); Tenorio-Lezama 16049 (MEXU); Tenorio-Lezama 16378 (MEXU); Tenorio-Lezama 16578 (MEXU); Tenorio-Lezama 16610 (MEXU); Webster 15724 (MEXU); Webster 15762 (MEXU)].

Lasianthaea palmeri (Greenm.) K.M. Becker [McVaugh 16383 (MEXU); Rzedowski-Rotter 14319 (MEXU); Stuessy 3061 (MEXU); Téllez-Valdés 8973 (MEXU)].

Lasianthaea rosei (Greenm.) McVaugh [Flores-Franco 1014 (MEXU); Téllez-Valdés 12081 (MEXU); Tenorio-Lezama 16102 (MEXU)].

Lasianthaea seemannii (A. Gray) K.M. Becker [Benítez-Paredes 3709 (MEXU); TéllezValdés 9845 (MEXU); Téllez-Valdés 10799 (MEXU); Webster 15730 (MEXU)].

Lasianthaea zinnioides (A. Gray) K.M. Becker [Flores-Franco 2433 (MEXU); Flores-Franco 2618 (MEXU); Flores-Franco 4068 (MEXU)].

Melampodium americanum L. [Benítez-Paredes 3216 (MEXU); Benítez-Paredes 3296 (MEXU); Bravo-Bolaños 2123 (MEXU); Flores-Franco 1023 (MEXU); Flores-Franco 2648 (MEXU); Flores-Franco 2756 (MEXU); Flores-Franco 2816 (ENCB, MEXU, MICH, US, XAL); King 3699 (MEXU); Téllez-Valdés 12055 (MEXU); Tenorio-Lezama 16845 (MEXU); Villaseñor-Ríos 713 (MEXU)].

Melampodium appendiculatum B.L. Rob. [Téllez-Valdés 11950 (MEXU)].

Melampodium divaricatum (Rich.) DC. [Aguilar-Orozco 357 (MEXU, UAN); Benítez-Paredes 3212 (MEXU); Benítez-Paredes 3215 (MEXU); Benítez-Paredes 3237 (MEXU); BenítezParedes 3248 (MEXU); Benítez-Paredes 3262 (MEXU); Benítez-Paredes 3569 (MEXU); Bravo-Bolaños 2067 (MEXU); Bravo-Bolaños 2145 (MEXU); Calzada 18584 (ENCB, MEXU, MICH, US); Calzada 18979 (EBCH, ENCB, FCME, MEXU, MICH, MO, US, XAL); Cronquist 9591 (MEXU); Flores-Franco 1268 (MEXU); Flores-Franco 1734 (MEXU); Flores-Franco 3704 (MEXU); Flores-Franco 3722 (MEXU); Flores-Franco 3816 (MEXU); Flores-Franco 3845 (MEXU); Flores-Franco 4115 (MEXU); Flores-Franco 4252 (MEXU); Flores-Franco 4308 (ENCB, MEXU, MICH, MO); García-Pérez 912 (MEXU); González 550 (MEXU); Hernández-Magaña 9699 (MEXU); King 3665 (MEXU); King 3674 (MEXU); King 3678 (MEXU); King 3687 (MEXU); King 3690 (MEXU); King 3691 (MEXU); King 3695 (MEXU); King 3696 (MEXU); King 3701 (MEXU); Norris 13684 (MEXU); RamírezRodríguez 586 (MEXU); Soule 2702 (MEXU, TEX); Soule 2712 (MEXU, TEX); TéllezValdés 9064 (MEXU); Téllez-Valdés 9097 (MEXU); Téllez-Valdés 10667 (MEXU); TéllezValdés 11160 (MEXU); Téllez-Valdés 12075 (MEXU); Téllez-Valdés 12472 (MEXU); Téllez-Valdés 12717 (MEXU); Téllez-Valdés 12794 (MEXU); Tenorio-Lezama 16299 (MEXU)]. 
Melampodium gracile Less. [Benítez-Paredes 3313 (MEXU); Bravo-Bolaños 2092 (MEXU)]. Melampodium longifolium Cerv. ex Cav. [Téllez-Valdés 9819 (MEXU)].

Melampodium microcephalum Less. [Benítez-Paredes 3261 (MEXU); Benítez-Paredes 3895 (MEXU); Flores-Franco 2699 (ENCB, MEXU, MICH, US); Flores-Franco 2708 (ENCB, MEXU, MICH, MO, UAMIZ, US, XAL); Ramírez-Rodríguez 581 (MEXU); Téllez-Valdés 12315 (MEXU)].

Melampodium perfoliatum (Cav.) Kunth [Flores-Franco 1356 (MEXU); Flores-Franco 1412 (MEXU); Flores-Franco 4407 (MEXU); Tenorio-Lezama 16412 (MEXU); Tenorio-Lezama 16468 (MEXU)].

Melampodium rosei B.L. Rob. [Colunga 3 (MEXU); Téllez-Valdés 11987 (MEXU)].

Melampodium sericeum Lag. [Bravo-Bolaños 2056 (MEXU)].

Melampodium tenellum Hook. \& Arn. [King 3703 (MEXU); King 3704 (MEXU); King 3705 (MEXU); King 3706 (MEXU); Ramírez-Rodríguez 469 (MEXU)].

Melampodium tepicense B.L. Rob. [Flores-Franco 2837 (ENCB, MEXU, MICH); FloresFranco 3814 (MEXU); Flores-Franco 4256 (MEXU); Stuessy 4118 (MEXU); Téllez-Valdés 9122 (MEXU); Téllez-Valdés 9730 (MEXU); Téllez-Valdés 9907 (MEXU); Téllez-Valdés 12859 (MEXU)].

Melanthera nivea (L.) Small [Téllez-Valdés 10348 (MEXU); Téllez-Valdés 11848 (MEXU)].

Mexianthus mexicanus B.L. Rob. [Flores-Franco 2393 (MEXU); Tenorio-Lezama 16719 (MEXU); Tenorio-Lezama 16819 (MEXU); Tenorio-Lezama 16825 (MEXU)].

Microspermum michoacanum (R.M. King) B.L. Turner [(Citado por Turner, 1997)].

Mikania cordifolia (L. f.) Willd. [Maltby 15 (Citado por McVaugh, 1984); McVaugh 23546 (Citado por McVaugh, 1984)]

Mikania micrantha Kunth [Téllez-Valdés 9438 (MEXU); Téllez-Valdés 12491 (MEXU)].

Milleria quinqueflora L. [Benítez-Paredes 3500 (MEXU); Flores-Franco 2633 (MEXU); McVaugh 16358 (MEXU); Stuessy 4122 (MEXU); Téllez-Valdés 12126 (MEXU)].

Montanoa andersonii McVaugh [Flores-Franco 3938 (MEXU); Tenorio-Lezama 16818 (MEXU, RSA)].

Montanoa bipinnatifida (Kunth) K. Koch [Flores-Franco 3141 (MEXU, MICH); Téllez-Valdés 9559 (MEXU)].

Montanoa karwinskii DC. [Calzada 18939 (ENCB, MEXU, MICH, MO); Flores-Franco 1288 (MEXU); Flores-Franco 1526 (MEXU); Flores-Franco 1680 (MEXU); Flores-Franco 4265 (MEXU); Flores-Franco 4267 (EBCH, ENCB, MEXU, MICH, MO, US, XAL); Téllez-Valdés 12521 (MEXU); Téllez-Valdés 12535 (MEXU); Téllez-Valdés 12988 (EBCH, ENCB, FCME, MEXU, MICH, MO, US, XAL); Tenorio-Lezama 16044 (MEXU); Tenorio-Lezama 16382 (MEXU); Tenorio-Lezama 16579 (MEXU)].

Montanoa leucantha (Lag. \& Segura) S.F. Blake subsp. leucantha [Flores-Franco 1703 (MEXU)].

Montanoa tomentosa Cerv. subsp. rosei (Rose ex B.L. Rob. \& Greenm.) V.A. Funk [TéllezValdés 4159 (MEXU)]. 
Onoseris onoseroides (Kunth) B.L. Rob. [Flores-Franco 3460 (MEXU, MICH); Téllez-Valdés 9045 (MEXU); Téllez-Valdés 10031 (MEXU)].

Otopappus microcephalus S.F. Blake [Flores-Franco 1311 (MEXU); Téllez-Valdés 9198 (MEXU); Tenorio-Lezama 16347 (MEXU)].

Otopappus tequilanus (A. Gray) B.L. Rob. [Calzada 18865 (MEXU); Cronquist 11186 (MEXU, NY); Cronquist 11189 (MEXU, NY); Flores-Franco 3775 (MEXU); Ramírez-Rodríguez 985 (MEXU); Vega-Ruiz 205 (FCME, MEXU)].

Oxypappus scaber Benth. [Flores-Franco 1901 (MEXU); Téllez-Valdés 9419 (MEXU); Tenorio-Lezama 15592 (MEXU); Tenorio-Lezama 16966 (MEXU)].

Packera bellidifolia (Kunth) W.A. Weber \& A. Löve [Diguet s.n. (Citado por McVaugh, 1984)]. Packera rosei (Greenm.) W.A. Weber \& A. Löve [Rose 2157 (Citado por McVaugh, 1984)].

Parthenium bipinnatifidum (Ortega) Rollins [Téllez-Valdés 9555 (MEXU); Téllez-Valdés 10239 (MEXU)].

Parthenium hysterophorus L. [Aguilar-Orozco 361 (MEXU, UAN); Flores-Franco 1563 (MEXU); Flores-Franco 3437 (MEXU); Gentry 11049 (MEXU); Téllez-Valdés 11289 (MEXU)].

Pectis diffusa Hook. \& Arn. [Flores-Franco 1600 (MEXU)].

Pectis multiflosculosa (DC.) Sch. Bip. [Chiang-Cabrera 1034 (MEXU); Téllez-Valdés 9257 (MEXU)].

Pectis prostrata Cav. [Keil 8978 (MEXU); Téllez-Valdés 10997 (MEXU)].

Pectis purpurea Brandegee var. lancifolia (Greenm.) D.J. Keil [Rose 1893 (GH, US)].

Pectis repens Brandegee [Feddema 1216 (Citado por McVaugh, 1984)].

Pectis uniaristata DC. var. uniaristata [Flores-Franco 1600 (MEXU, RSA)].

Pectis uniaristata DC. var. jangadensis (S. Moore) D.J. Keil [González-Ortega 1083 (MEXU)].

Perityle microglossa Benth. var. microglossa [Benítez-Paredes 2993 (MEXU); Bravo-Bolaños 2148 (MEXU); Chiang-Cabrera 1115 (MEXU); Flores-Franco 1900 (MEXU); McVaugh 12147 (MEXU); Téllez-Valdés 4146 (MEXU); Téllez-Valdés 8265 (MEXU); Téllez-Valdés 9364 (MEXU); Téllez-Valdés 11480 (MEXU); Téllez-Valdés 11487 (MEXU)].

Perityle microglossa Benth. var. saxosa (Brandegee) A.M. Powell [Flores-Franco 2023 (MEXU); Flores-Franco 2391 (MEXU); Ramírez-Rodríguez 867 (MEXU); Tenorio-Lezama 15622 (MEXU)].

Perityle rosei Greenm. [Téllez-Valdés 10623 (MEXU)].

Perityle trichodonta S.F. Blake [Flores-Franco 2458 (MEXU); Ramírez-Rodríguez 807 (MEXU); Téllez-Valdés 11416 (MEXU); Téllez-Valdés 12210 (MEXU); Tenorio-Lezama 16176 (MEXU)].

Perymenium buphtalmoides DC. var. buphtalmoides [Norris 14236 (Citado por McVaugh, 1984)].

Perymenium buphtalmoides DC. var. diguetii (McVaugh) B.L. Turner [Benítez-Paredes 3439 (MEXU); Flores-Franco 2259 (MEXU)].

Perymenium buphtalmoides DC. var. occidentale McVaugh [McVaugh 15147 (MEXU)]. 
Perymenium buphtalmoides DC. var. tenellum (A. Gray) McVaugh [Norris 14686 (Citado por McVaugh, 1984)].

Perymenium oxycarphum S.F. Blake [Flores-Franco 2893 (MEXU); Flores-Franco 4542 (MEXU); González-Flores 810 (MEXU, UAN); Téllez-Valdés 13016 (MEXU); TéllezValdés 13025 (MEXU)].

Perymenium pringlei B.L. Rob. \& Greenm. var. croceum (B.L. Rob. \& Greenm.) Fay [FloresFranco 2147 (ENCB, MEXU, MICH, MO, XAL); Ramírez-Rodríguez 978 (ENCB, MEXU, MICH, MO, XAL); Téllez-Valdés 10684 (ENCB, MEXU, MICH); Téllez-Valdés 12201 (ENCB, MEXU, MICH, MO); Tenorio-Lezama 16469 (ENCB, MEXU, MICH, MO)].

Perymenium stenophyllum S.F. Blake [Flores-Franco 1413 (MEXU)].

Perymenium uxoris McVaugh [Téllez-Valdés 11679 (MEXU); Tenorio-Lezama 16934 (MEXU)].

Pinaropappus diguetii McVaugh [Diguet s.n. (Citado por McVaugh, 1984); Rose 2094 (Citado por McVaugh, 1984)].

Pippenalia delphinifolia (Rydb.) McVaugh [Ramírez-Rodríguez 923 (MEXU); TenorioLezama 15955 (MEXU)].

Piqueria triflora Hemsl. [Calzada 19084 (MEXU); Calzada 19238 (EBCH, ENCB, MEXU, MICH, MO, US, XAL); Flores-Franco 1927 (MEXU); Flores-Franco 2353 (MEXU); FloresFranco 2507 (MEXU); Flores-Franco 3990 (MEXU); Flores-Franco 4027 (ENCB, MEXU, MICH); Flores-Franco 4029 (MEXU); González-Flores 1331 (MEXU, UAN); Téllez-Valdés 9584 (MEXU); Téllez-Valdés 9808 (MEXU); Téllez-Valdés 9933 (MEXU); Téllez-Valdés 9997 (MEXU); Téllez-Valdés 11110 (MEXU, MICH); Téllez-Valdés 12318 (ENCB, MEXU, $\mathrm{MICH}$ ); Téllez-Valdés 12631 (EBCH, ENCB, FCME, MEXU, MICH, MO, US, XAL); Tenorio-Lezama 16783 (MEXU); Tenorio-Lezama 17010 (ENCB, MEXU, MICH, MO, $\mathrm{XAL})]$.

Piqueria trinervia Cav. [Croat 45142 (MEXU); Miller 3216 (MEXU, MO); Téllez-Valdés 11786 (MEXU)].

Pittocaulon praecox (L.) H. Rob. \& Brettell var. morelense (Miranda) H. Rob. \& Brettell [McVaugh 14911 (MEXU); Téllez-Valdés 12829 (MEXU)].

Pittocaulon velatum (Greenm.) H. Rob. \& Brettell var. velatum [Gregg 948 (GH, MO); McVaugh 12054 (MICH); McVaugh 14911 (MICH)].

Pluchea odorata (L.) Cass. [González-Ortega 5538 (MEXU); King 3697 (MEXU)].

Pluchea salicifolia (Mill.) S.F. Blake [Téllez-Valdés 9439 (MEXU); Téllez-Valdés 10253 (MEXU); Téllez-Valdés 11873 (MEXU)].

Pluchea symphytifolia (Mill.) Gillis [Maltby 24 (Citado por McVaugh, 1984)].

Porophyllum coloratum (Kunth) DC. var. coloratum [Aguilar-Orozco 325 (MEXU, UAN); Aguilar-Orozco 402 (MEXU, UAN); Benítez-Paredes 3605 (MEXU); Flores-Franco 3959 (MEXU); Solís, I. 654 (MEXU); Téllez-Valdés 9546 (MEXU); Téllez-Valdés 9824 (MEXU); Tenorio-Lezama 16790 (MEXU)].

Porophyllum coloratum (Kunth) DC. var. obtusifolium (DC.) McVaugh [Téllez-Valdés 11373 (MEXU); Téllez-Valdés 11797 (MEXU)]. 
Porophyllum linaria (Cav.) DC. [Téllez-Valdés 11056 (MEXU)].

Porophyllum lindenii Sch. Bip. [Mexia 636 (Citado por McVaugh, 1984)].

Porophyllum macrocephalum DC. [Flores-Franco 1044 (MEXU); Flores-Franco 1055

(MEXU); Téllez-Valdés 11103 (MEXU); Villaseñor-Ríos 715 (MEXU)].

Porophyllum punctatum (Mill.) S.F. Blake [Aguilar-Orozco 380 (MEXU, UAN); Benítez-

Paredes 3959 (MEXU); Chiang-Cabrera 1095 (MEXU); Flores-Franco 879 (MEXU, RSA);

Flores-Franco 2726 (MEXU, MICH); Flores-Franco 4006 (MEXU); Téllez-Valdés 10084

(MEXU); Téllez-Valdés 11162 (MEXU)].

Porophyllum viridiflorum (Kunth) DC. [Benítez-Paredes 3280 (MEXU); Téllez-Valdés 11001

(MEXU)].

Psacalium cervinum (Rydb.) H. Rob. \& Brettell [McVaugh 15313 (MEXU)].

Psacalium eriocarpum (S.F. Blake) S.F. Blake [González-Flores 1314 (MEXU)].

Psacalium filicifolium (Rydb.) H. Rob. \& Brettell [Rose 2101 (US)].

Psacalium laxiflorum Benth. [Norris 14577 (Citado por McVaugh, 1984)].

Psacalium megaphyllum (B.L. Rob. \& Greenm.) Rydb. [Flores-Franco 4043 (MEXU); TéllezValdés 10572 (MEXU); Tenorio-Lezama 16800 (MEXU)].

Psacalium palmeri (Greene) H. Rob. \& Brettell [Breedlove 61538 (MEXU); Calzada 18721

(ENCB, MEXU, MICH); Flores-Franco 1155 (MEXU); Téllez-Valdés 9337 (MEXU); Téllez-

Valdés 9934 (MEXU); Téllez-Valdés 11576 (MEXU); Téllez-Valdés 12059 (MEXU);

Tenorio-Lezama 16147 (MEXU)].

Psacalium peltigerum (B.L. Rob. \& Seaton) Rydb. var. peltigerum [Flores-Franco 2073 (MEXU)].

Psacalium poculiferum (S. Watson) Rydb. [McVaugh 16406 (MEXU); Téllez-Valdés 10680 (MEXU)].

Psacalium pringlei (S. Watson) H. Rob. \& Brettell [Calzada 18700 (MEXU); Calzada 18843 (MEXU, MICH); Téllez-Valdés 9567 (MEXU); Téllez-Valdés 9799 (MEXU); TenorioLezama 16669 (MEXU); Tenorio-Lezama 16894 (MEXU)].

Psacalium sinuatum (Cerv.) H. Rob. \& Brettell [Breedlove 61424 (MEXU)].

Pseudelephantopus spicatus (Aubl.) Rohr [Aguilar-Orozco 319 (MEXU, UAN); AguilarOrozco 365 (MEXU), UAN; Aguilar-Orozco 525 (MEXU, UAN); Aguilar-Orozco 531 (MEXU, UAN); Benítez-Paredes 2974 (MEXU); Téllez-Valdés 10234 (MEXU); TéllezValdés 11149 (MEXU); Téllez-Valdés 11315 (MEXU)].

Pseudoconyza viscosa (Mill.) D’Arcy var. lyrata (Kunth) D’Arcy [Téllez-Valdés 10500 (MEXU)].

Pseudognaphalium arizonicum (A. Gray) A. Anderb. [Téllez-Valdés 9443 (MEXU)].

Pseudognaphalium attenuatum (DC.) A. Anderb. [Flores-Franco 1611 (MEXU); FloresFranco 2437 (MEXU); Flores-Franco 3279 (ENCB, MEXU, MICH, MO); Flores-Franco 3279 (MEXU); Nuñez-Pérez 1160 (MEXU, UAN); Téllez-Valdés 9392 (MEXU); TéllezValdés 9587 (MEXU); Téllez-Valdés 11410 (MEXU); Téllez-Valdés 11474 (MEXU); Téllez-Valdés 12594 (EBCH, ENCB, FCME, MEXU, MICH, MO, US, XAL); Téllez-Valdés 12632 (MEXU)].

Pseudognaphalium bourgovii (A. Gray) A. Anderb. [Calzada 18917 (EBCH, ENCB, MEXU, $\mathrm{MICH}, \mathrm{MO}$, US, XAL); Flores-Franco 2548 (MEXU)]. 
Pseudognaphalium canescens (DC.) A. Anderb. [Aguilar-Orozco 496 (MEXU, UAN); NuñezPérez 1160 (FCME, MEXU); Téllez-Valdés 10090 (MEXU)].

Pseudognaphalium chartaceum (Greenm.) A. Anderb. [Calzada 19087 (MEXU); TéllezValdés 9601 (MEXU)].

Pseudognaphalium jaliscense (Greenm.) A. Anderb. [Calzada 18765 (ENCB, MEXU, MICH, MO, UAMIZ, US, XAL); Ramírez-Rodríguez 945 (MEXU); Téllez-Valdés 8997 (MEXU); Téllez-Valdés 9223 (MEXU); Tenorio-Lezama 15658 (MEXU)].

Pseudognaphalium leucocephalum (A. Gray) A. Anderb. [Flores-Franco 1420 (MEXU); Téllez-Valdés 11509 (MEXU); Téllez-Valdés 11565 (MEXU)].

Pseudognaphalium roseum (Kunth) A. Anderb. [Solís 680 (MEXU)].

Pseudognaphalium semilanatum (DC.) A. Anderb. [Flores-Franco 3270 (MEXU, MICH)].

Pseudognaphalium stramineum (Kunth) A. Anderb. [González-Ortega 5543 (MEXU)].

Pseudognaphalium viscosum (Kunth) A. Anderb. [Benítez-Paredes 3773 (MEXU); FloresFranco 2254 (MEXU)].

Roldana albonervia (Greenm.) H. Rob. \& Brettell [Téllez-Valdés 9125 (MEXU)].

Roldana angulifolia (DC.) H. Rob. \& Brettell [Santana-Michel 2412 (MEXU)].

Roldana chapalensis (S. Watson) H. Rob. \& Brettell [Calzada 19090 (MEXU); Flores-Franco 3263 (ENCB, MEXU, MICH, MO)].

Roldana guadalajarensis (B.L. Rob.) H. Rob. \& Brettell [Flores-Franco 1164 (MEXU); FloresFranco 1451 (MEXU); Téllez-Valdés 12127 (MEXU); Tenorio-Lezama 16003 (MEXU); Tenorio-Lezama 16518 (MEXU)].

Roldana hartwegii (Benth.) H. Rob. \& Brettell [Flores-Franco 1435 (MEXU); Flores-Franco 3169 (ENCB, MEXU, MICH, MO); Flores-Franco 3190 (EBCH, ENCB, MEXU, MICH, MO, US, XAL); Flores-Franco 3251 (ENCB, MEXU, MICH, MO); Flores-Franco 4032 (MEXU); Flores-Franco 4374 (MEXU); Flores-Franco 4461 (MEXU); Flores-Franco 4536 (MEXU); Téllez-Valdés 9518 (MEXU); Téllez-Valdés 11590 (MEXU); Tenorio-Lezama 16502 (ENCB, MEXU, MICH); Tenorio-Lezama 16909 (MEXU); Tenorio-Lezama 16981 (ENCB, MEXU, MICH, MO)].

Roldana sessilifolia (Hook. \& Arn.) H. Rob. \& Brettell [Aguilar-Orozco 335 (MEXU, UAN); Aguilar-Orozco 466 (MEXU, UAN); Flores-Franco 2906 (MEXU, MICH); Flores-Franco 3257 (ENCB, MEXU, MICH, MO, XAL); Flores-Franco 3273 (MEXU); Téllez-Valdés 9424 (MEXU); Téllez-Valdés 9531 (MEXU); Téllez-Valdés 9780 (MEXU); Téllez-Valdés 11591 (MEXU); Téllez-Valdés 12134 (MEXU); Téllez-Valdés 12226 (MEXU); Téllez-Valdés 13023 (MEXU); Tenorio-Lezama 16744 (MEXU); Tenorio-Lezama 16893 (MEXU)].

Roldana subpeltata (Sch. Bip.) H. Rob. \& Brettell [Téllez-Valdés 12626 (MEXU)].

Roldana suffulta (Greenm.) H. Rob. \& Brettell [Flores-Franco 4120 (MEXU); Flores-Franco 4188 (MEXU, MICH); Flores-Franco 4253 (MEXU); Flores-Franco 4298 (ENCB, MEXU, MICH); Téllez-Valdés 9470 (MEXU); Téllez-Valdés 12412 (MEXU)].

Rumfordia floribunda DC. var. floribunda [Flores-Franco 3504 (MEXU, UAN)].

Rumfordia floribunda DC. var. jaliscensis R.W. Sanders [Blanco-Correa 23 (MEXU, UAN); Calzada 19173 (EBCH, ENCB, FCME, MEXU, MICH, MO, US, XAL); Flores-Franco 1925 (MEXU); Flores-Franco 2553 (MEXU); Flores-Franco 3686 (ENCB, MEXU, MICH); González-Flores 569 (MEXU); González-Flores 579 (MEXU); González-Flores 647 (MEXU); Madrigal-Sánchez 2512 (MEXU); Magaña-Rueda 84 (MEXU); McVaugh 12121 
(MEXU); Téllez-Valdés 10025 (MEXU); Téllez-Valdés 10422 (MEXU); Téllez-Valdés 11695 (MEXU); Téllez-Valdés 12563 (MEXU); Tenorio-Lezama 15614 (MEXU)].

Salmea palmeri S. Watson [Flores-Franco 1675 (MEXU)].

Schkuhria anthemoidea (DC.) Coulter var. anthemoidea [Keil 15086 (MEXU)].

Schkuhria pinnata (Lam.) Kuntze var. guatemalensis (Rydb.) McVaugh [Feddema 1414

(Citado por McVaugh, 1984); Mexia 657 (Citado por McVaugh, 1984)].

Schkuhria pinnata (Lam.) Kuntze var. virgata (Llave) Heiser [Breedlove 44493 (MEXU);

Ferris 5857 (MEXU); Téllez-Valdés 9347 (MEXU)].

Schkuhria schkuhrioides (Link \& Otto) Thell. [Téllez-Valdés 11513 (MEXU)].

Sclerocarpus divaricatus (Benth.) Hemsl. [Benítez-Paredes 3389 (MEXU); Benítez-Paredes 3415 (MEXU); Benítez-Paredes 3439 (MEXU); Benítez-Paredes 3465 (MEXU); BenítezParedes 3517 (MEXU); Benítez-Paredes 3678 (MEXU); Benítez-Paredes 3844 (MEXU); Calzada 18655 (MEXU); Colunga 8 (MEXU); Cronquist 9596 (MEXU); Flores-Franco 973 (MEXU); Flores-Franco 1050 (MEXU); Flores-Franco 2662 (MEXU); Flores-Franco 2700 (ENCB, MEXU, MICH, US); Villaseñor-Ríos 710 (MEXU)].

Sclerocarpus sessilifolius Greenm. [Calzada 18825 (MEXU); Flores-Franco 3912 (MEXU); McVaugh 16587 (MEXU); Téllez-Valdés 8971 (MEXU); Téllez-Valdés 12064 (MEXU); Tenorio-Lezama 16210 (MEXU)].

Sclerocarpus uniserialis (Hook.) Benth. \& Hook. f. ex Hemsl. var. frutescens (Brandegee) Feddema [King 3673 (MEXU); Téllez-Valdés 9838 (MEXU); Téllez-Valdés 11460 (MEXU)].

Senecio carlomasonii B.L. Turner \& T.M. Barkley [McVaugh 652 (LL, MICH)].

Sigesbeckia jorullensis Kunth [Flores-Franco 3225 (ENCB, MEXU, MICH)].

Simsia annectens S.F. Blake var. annectens [Ramírez-Rodríguez 864 (MEXU)].

Simsia foetida (Cav.) S.F. Blake var. foetida [Gentry 19532 (Citado por McVaugh, 1984)]. Simsia sanguinea A. Gray [Flores-Franco 1667 (MEXU); Flores-Franco 1670 (MEXU); Tenorio-Lezama 16065 (MEXU)].

Simsia setosa S.F. Blake [Colunga 21 (MEXU); Spooner 2528 (MEXU); Villaseñor-Ríos 706 (MEXU)].

Sinclairia angustissima (A. Gray) B.L. Turner [Rose 3406 (Citado por McVaugh, 1984)]. Sinclairia cervina (B.L. Rob.) B.L. Turner [McVaugh 16583 (MEXU)].

Sinclairia gentryi (H. Rob.) B.L. Turner [Gentry 11030 (MEXU)].

Sinclairia glabra (Hemsl.) Rydb. var. hypoleuca (Greenm.) B.L. Turner [Téllez-Valdés 9883 (MEXU)].

Sinclairia palmeri (A. Gray) B.L. Turner [Tenorio-Lezama 16037 (MEXU); Tenorio-Lezama 16169 (MEXU)].

Sinclairia pringlei (B.L. Rob. \& Greenm.) H. Rob. \& Brettell [Flores-Franco 1332 (MEXU); Téllez-Valdés 9334 (MEXU); Téllez-Valdés 9942 (MEXU); Téllez-Valdés 12145 (MEXU);

Tenorio-Lezama 16053 (MEXU); Tenorio-Lezama 16366 (MEXU)].

Sinclairia similis (McVaugh) H. Rob. \& Brettell [Cronquist 10794 (MEXU)]. 
Smallanthus apus (S.F. Blake) H. Rob. [Rose 2079 (NY, US)].

Smallanthus maculatus (Cav.) H. Rob. var. maculatus [Calzada 18750 (ENCB, MEXU, MICH, US); Calzada 18935 (MEXU); Calzada 19057 (MEXU); Flores-Franco 3836 (ENCB, MEXU, MICH, MO, XAL); Flores-Franco 3849 (MEXU); Flores-Franco 3921 (MEXU); Flores-Franco 4121 (MEXU); Flores-Franco 4213 (MEXU); Flores-Franco 4477 (MEXU); Téllez-Valdés 9218 (MEXU)].

Sonchus oleraceus L. [Flores-Franco 4345 (MEXU); Téllez-Valdés 9491 (MEXU); TéllezValdés 11237 (MEXU); Téllez-Valdés 12559 (MEXU)].

Spilanthes urens Jacq. [Webster 17098 (Citado por McVaugh, 1984)].

Stevia alatipes B.L. Rob. [Bauml 1096 (Citado por McVaugh, 1984)].

Stevia anadenotricha (B.L. Rob.) Grashoff [Flores-Franco 2360 (MEXU); Ramírez-Rodríguez 704 (MEXU)].

Stevia aschenborniana Sch. Bip. [McVaugh 839 (Citado por McVaugh, 1984)].

Stevia caracasana DC. [Téllez-Valdés 9602 (MEXU)].

Stevia dictyophylla B.L. Rob. [(Citado por Turner, 1997)].

Stevia elatior Kunth [Téllez-Valdés 9398 (MEXU)].

Stevia glandulosa Hook. \& Arn. var. glandulosa [Madrigal-Sánchez 2525 (MEXU); Téllez-

Valdés 8232 (MEXU); Téllez-Valdés 9578 (MEXU)].

Stevia jaliscensis B.L. Rob. [Flores-Franco 2473 (MEXU); Flores-Franco 3297 (MEXU),

Ramírez-Rodríguez 789 (MEXU)].

Stevia lasioclada Grashoff [Paray 2710 (ENCB)].

Stevia lucida Lag. var. lucida [Téllez-Valdés 11600 (MEXU)].

Stevia micradenia B.L. Rob. [González-Flores 1331 (MEXU); Téllez-Valdés 11601 (MEXU);

Tenorio-Lezama 16753 (MEXU); Tenorio-Lezama 16782 (MEXU)].

Stevia micrantha Lag. [Mexia 897 (Citado por McVaugh, 1984)].

Stevia myricoides McVaugh [Flores-Franco 2546 (MEXU); McVaugh 12126 (MEXU); Téllez-

Valdés 10419 (MEXU, MICH); Tenorio-Lezama 15695 (MEXU)].

Stevia origanoides Kunth [Ferris 5823 (MEXU); Flores-Franco 1693 (MEXU); Flores-Franco

4431 (ENCB, MEXU, MICH); Flores-Franco 4490 (MEXU); González-Flores 1310

(MEXU, UAN); Téllez-Valdés 12525 (MEXU)].

Stevia ovalis (B.L. Rob.) B.L. Rob. [Tenorio-Lezama 16743 (MEXU)].

Stevia ovata Willd. var. ovata [Calzada 18840 (ENCB, MEXU, MICH, MO); Flores-Franco

1643 (MEXU); Flores-Franco 3162 (ENCB, MEXU, MICH, MO); Flores-Franco 3198

(ENCB, MEXU, MICH, MO); Flores-Franco 3977 (MEXU); Flores-Franco 4029 (MEXU);

Flores-Franco 4355 (MEXU); Flores-Franco 4387 (MEXU); González-Flores 950 (MEXU,

UAN); Téllez-Valdés 9937 (MEXU); Téllez-Valdés 11492 (MEXU); Téllez-Valdés 11499

(MEXU); Téllez-Valdés 11560 (MEXU); Téllez-Valdés 12999 (MEXU); Téllez-Valdés

13035 (ENCB, MEXU, MICH); Webster 15741 (MEXU); Webster 15830 (MEXU)].

Stevia rosei B.L. Rob. [Flores-Franco 1347 (MEXU); Tenorio-Lezama 16404 (MEXU)].

Stevia seemannii Sch. Bip. [Seemann 2041 (GH, K, P)].

Stevia serrata Cav. var. serrata [Aguilar-Orozco 415 (MEXU, UAN); Blanco-Correa 68

(MEXU, UAN); Breedlove 44585 (MEXU); Flores-Franco 1154 (MEXU); Flores-Franco

1422 (MEXU); Flores-Franco 4003 (MEXU); Flores-Franco 4518 (MEXU, MICH); 
González-Flores 950 (MEXU, UAN);Téllez-Valdés 8996 (MEXU); Téllez-Valdés 11545 (MEXU); Tenorio-Lezama 16539 (MEXU)].

Stevia subpubescens Lag. var. opaca (Sch. Bip.) B.L. Rob. [Flores-Franco 1724 (MEXU); Tenorio-Lezama 15964 (MEXU); Flores-Franco 3200 (ENCB, MEXU, MICH, MO, XAL)]. Stevia villaregalis McVaugh [Ramírez-Rodríguez 1104 (MEXU)].

Stevia viscida Kunth [Breedlove 44479 (MEXU); Calzada 18860 (MEXU, MICH); FloresFranco 1421 (MEXU); Flores-Franco 2368 (MEXU); González-Flores 1317 (MEXU); Téllez-Valdés 11511 (MEXU); Tenorio-Lezama 16538 (MEXU); Tenorio-Lezama 16671 (MEXU)].

Steviopsis adenosperma (Sch. Bip.) B.L. Turner [Seemann 2037 (K, P)].

Steviopsis dryophila (B.L. Rob.) B.L. Turner [Calzada 18814 (MEXU); Croat 45238 (MEXU); Flores-Franco 2442 (MEXU); McVaugh 25760 (MEXU, MICH)].

Steviopsis rapunculoides (DC.) R.M. King \& H. Rob. [(Citado por Turner, 1997)].

Synedrella nodiflora (L.) Gaertn. [Benítez-Paredes 2987 (MEXU); Benítez-Paredes 3619 (MEXU); Benítez-Paredes 3679 (MEXU); Benítez-Paredes 3783 (MEXU)].

Tagetes erecta L. [Bravo-Bolaños 2077 (MEXU); Bravo-Bolaños 2087 (MEXU); BravoBolaños 2125 (MEXU); Nuñez-Pérez 1159 (FCME, MEXU, UAN); Nuñez-Pérez 1163 (FCME, MEXU, UAN); Téllez-Valdés 11006 (MEXU); Tenorio-Lezama 16112 (MEXU); Tenorio-Lezama 16927 (MEXU)].

Tagetes filifolia Lag. [Aguilar-Orozco 314 (MEXU, UAN); Aguilar-Orozco 421 (MEXU, UAN); Bauml 1088 (MEXU); Bravo-Hollis 9311 (MEXU); Breedlove 44480 (MEXU); FloresFranco 1170 (MEXU); Flores-Franco 1344 (MEXU); Flores-Franco 3171 (ENCB, MEXU, $\mathrm{MICH}, \mathrm{MO}$ ); Flores-Franco 3919 (MEXU, MICH); Flores-Franco 4047 (ENCB, MEXU, $\mathrm{MICH}, \mathrm{MO}$ ); Flores-Franco 4404 (MEXU); González-Flores 580 (MEXU); Téllez-Valdés 11062 (MEXU); Téllez-Valdés 11471 (MEXU); Téllez-Valdés 13002 (MEXU); TenorioLezama 16343 (MEXU)].

Tagetes hartwegii Greenm. [(Citado por Turner, 1996)].

Tagetes lucida Cav. [Breedlove 44599 (MEXU); Reveal 4085 (MEXU); Téllez-Valdés 8978 (MEXU); Téllez-Valdés 10987 (MEXU)].

Tagetes persicifolius (Benth.) B.L. Turner [Flores-Franco 1135 (MEXU); Flores-Franco 1688 (MEXU); Téllez-Valdés 12256 (MEXU); Tenorio-Lezama 16731 (MEXU)].

Tagetes subulata Cerv. [Calzada 18835 (MEXU); Flores-Franco 1601 (MEXU); Téllez-Valdés 11789 (MEXU); Tenorio-Lezama 16663 (MEXU)].

Tagetes tenuifolia Cav. [Benítez-Paredes 3572 (MEXU); Calzada 18833 (MEXU); TéllezValdés 9360 (MEXU)].

Tagetes triradiata Greenm. [Flores-Franco 1394 (MEXU); Flores-Franco 1617 (MEXU); Tenorio-Lezama 16214 (MEXU); Tenorio-Lezama 16449 (MEXU)].

Thymophylla pentachaeta (DC.) Small var. belenidium (DC.) Strother [Matuda s.n. (MEXU)].

Tithonia calva Sch. Bip. var. lancifolia (B.L. Rob. \& Greenm.) McVaugh [Ramírez-Rodríguez 611 (MEXU); Téllez-Valdés 12202 (MEXU); Téllez-Valdés 12914 (ENCB, MEXU, MICH, $\mathrm{MO}, \mathrm{XAL})]$. 
Tithonia rotundifolia (Mill.) S.F. Blake [Benítez-Paredes 3389 (MEXU); Flores-Franco 1494 (MEXU); Flores-Franco 3019 (MEXU); Téllez-Valdés 12325 (MEXU); Téllez-Valdés 12518 (ENCB, MEXU, MICH, MO, US, XAL); Tenorio-Lezama 16548 (MEXU); TenorioLezama 16582 (MEXU)].

Tithonia tubiformis (Jacq.) Cass. [Flores-Franco 1529 (MEXU); Flores-Franco 1572 (MEXU); Flores-Franco 3115 (MEXU); Flores-Franco 4476 (MEXU); Téllez-Valdés 9594 (MEXU); Téllez-Valdés 11268 (MEXU); Téllez-Valdés 11385 (MEXU, MICH); Tenorio-Lezama 16876 (MEXU)].

Tridax coronopifolia (Kunth) Hemsl. [Tenorio-Lezama 16013 (MEXU)].

Tridax dubia Rose [Flores-Franco 907 (MEXU); King 3666 (MEXU); Stuessy 3064 (MEXU); Téllez-Valdés 11166 (MEXU); Téllez-Valdés 11173 (MEXU); Téllez-Valdés 12865 (MEXU)].

Tridax mexicana A.M. Powell [Flores-Franco 3435 (MEXU); Téllez-Valdés 9408 (MEXU); Téllez-Valdés 12063 (MEXU)].

Tridax platyphylla B.L. Rob. [Flores-Franco 2737 (ENCB, MEXU, MICH, US, XAL)].

Tridax procumbens L. [Calzada 18594 (MEXU); Calzada 18826 (MEXU); Calzada 19126 (MEXU); Flores-Franco 2128 (MEXU); Flores-Franco 3307 (MEXU); King 3680 (MEXU); King 3702 (MEXU); Téllez-Valdés 10257 (MEXU); Téllez-Valdés 11470 (MEXU)].

Trigonospermum melampodioides DC. [Calzada 18919 (ENCB, MEXU, MICH, MO, US, XAL); Flores-Franco 1389 (MEXU); Flores-Franco 3850 (MEXU); Flores-Franco 4072 (MEXU); Flores-Franco 4119 (MEXU); Flores-Franco 4388 (MEXU, MICH); Téllez-Valdés 9130 (MEXU); Téllez-Valdés 9478 (MEXU); Tenorio-Lezama 16445 (MEXU)].

Trixis angustifolia DC. [Aguilar-Orozco 494 (MEXU, UAN)].

Trixis hyposericea S. Watson [Arcadia-Alvarez 184 (MEXU); Calzada 19103 (MEXU); FloresFranco 1607 (MEXU); Flores-Franco 1765 (MEXU); Flores-Franco 1823 (MEXU); TéllezValdés 12441 (MEXU); Tenorio-Lezama 16627 (MEXU)].

Trixis inula Crantz [Benítez-Paredes 2988 (MEXU); Chiang-Cabrera 1185 (MEXU)].

Trixis michuacana Lex. var. longifolia (D. Don) C. Anderson [Aguilar-Orozco 321 (MEXU, UAN); Benítez-Paredes 3708 (MEXU); Bravo-Bolaños 2178 (MEXU); Flores-Franco 2561 (MEXU); González-Flores 701 (MEXU, UAN); Mayfield 1655 (MEXU, TEX); Téllez-Valdés 9592 (MEXU); Téllez-Valdés 10096 (MEXU)].

Trixis pterocaulis B.L. Rob. \& Greenm. [Chávez s.n. (ENCB, MEXU); Chiang-Cabrera 1098 (MEXU); Téllez-Valdés 4134 (MEXU)].

Verbesina angustifolia (Benth.) S.F. Blake [Calzada 18916 (MEXU); Calzada 19051 (ENCB, MEXU, MICH); Flores-Franco 1715 (MEXU); Flores-Franco 2378 (MEXU); Flores-Franco 2450 (MEXU); Flores-Franco 3218 (ENCB, MEXU, MICH, MO); Flores-Franco 3250 (MEXU); Flores-Franco 4111 (MEXU); Flores-Franco 4526 (MEXU); Ramírez-Rodríguez 723 (MEXU); Ramírez-Rodríguez 724 (MEXU); Tenorio-Lezama 16453 (MEXU); TenorioLezama 16764 (MEXU); Tenorio-Lezama 17014 (ENCB, MEXU, MICH, MO, XAL)].

Verbesina crocata (Cav.) Less. [Benítez-Paredes 3508 (MEXU); Bravo-Bolaños 2136 (MEXU); Téllez-Valdés 8259 (MEXU); Tenorio-Lezama 16515 (MEXU, MICH)].

Verbesina fastigiata B.L. Rob. \& Greenm. [Flores-Franco 1822 (MEXU); Téllez-Valdés 10093 (MEXU); Tenorio-Lezama 16713 (MEXU)]. 
Verbesina fusiformis McVaugh [Flores-Franco 3219 (EBCH, ENCB, FCME, MEXU, MICH, $\mathrm{MO}$, US, XAL)].

Verbesina greenmanii Urb. [Flores-Franco 3170 (ENCB, MEXU, MICH, MO); Flores-Franco 4444 (ENCB, MEXU, MICH); Flores-Franco 4459 (MEXU); Téllez-Valdés 9366 (MEXU); Téllez-Valdés 9575 (MEXU); Téllez-Valdés 13017 (MEXU); Tenorio-Lezama 17028 (MEXU)].

Verbesina hispida McVaugh [Flores-Franco 3714 (MEXU)].

Verbesina lottiana B.L. Turner \& Olsen [Tenorio-Lezama 15560 (MEXU)].

Verbesina madrensis Greenm. [Rose 3378 (US)].

Verbesina nayaritensis B.L. Turner [Solís 560 (TEX)].

Verbesina oncophora B.L. Rob. \& Seaton var. subhamata McVaugh [Flores-Franco 1398 (MEXU)].

Verbesina oxylepis S.F. Blake [Calzada 18758 (MEXU, MICH)].

Verbesina pantoptera S.F. Blake [Calzada 18902 (ENCB, MEXU, MICH, MO); Flores-Franco 2903 (MEXU); Flores-Franco 3797 (MEXU); Flores-Franco 4013 (MEXU); Flores-Franco 4035 (MEXU); Flores-Franco 4515 (MEXU); González-Flores 622 (MEXU, UAN); González-Flores 791 (MEXU, UAN); Téllez-Valdés 9152 (MEXU); Téllez-Valdés 9763 (MEXU); Tenorio-Lezama 16751 (MEXU)].

Verbesina parviflora (Kunth) S.F. Blake var. parviflora [Rose 3397 (Citado por McVaugh, 1984)].

Verbesina pedunculosa (DC.) B.L. Rob. [McVaugh 16428 (MEXU)].

Verbesina rosei B.L. Rob. \& Greenm. [Ramírez-Rodríguez 567 (MEXU)].

Verbesina sphaerocephala A. Gray var. dubia McVaugh [Calzada 18863 (MEXU)].

Verbesina sphaerocephala A. Gray var. sphaerocephala [Campos-Villanueva 5038 (MEXU, XAL); Flores-Franco 1331 (MEXU); Flores-Franco 1700 (MEXU); Flores-Franco 1833 (MEXU); Flores-Franco 3136 (ENCB, MEXU, MICH); Téllez-Valdés 9473 (MEXU); TéllezValdés 9571 (MEXU); Téllez-Valdés 11442 (MEXU); Tenorio-Lezama 16365 (MEXU)]. Verbesina tequilana J.R. Coleman [Téllez-Valdés 8949 (MEXU)].

Verbesina tetraptera (Ortega) A. Gray [Flores-Franco 1448 (MEXU)].

Verbesina vallartana B.L. Turner \& Olsen [Panero 2570 (MEXU)].

Vernonia bolleana Sch. Bip. [Seemann 1992 (K, P)].

Vernonia canescens Kunth [Chiang-Cabrera 1112 (MEXU)].

Vernonia deppeana Less. [Benítez-Paredes 3131 (MEXU); Benítez-Paredes 3758 (MEXU); Calzada 19142 (MEXU); Flores-Franco 3359 (MEXU); Flores-Franco 3512 (MEXU, UAN); McVaugh 12072 (MEXU); Téllez-Valdés 10479 (MEXU)].

Vernonia feddemae McVaugh [Feddema, C. 846A (MICH)].

Vernonia liatroides DC. subsp. ehrenbergiana (Sch. Bip.) S.B. Jones [Téllez-Valdés 11437 (MEXU)].

Vernonia liatroides DC. subsp. gentryi S.B. Jones [Aguilar-Orozco 494 (MEXU, UAN); Calzada 19213 (ENCB, MEXU, MICH, MO); Calzada 19222 (ENCB, MEXU, MICH); Flores-Franco 1696 (MEXU); Flores-Franco 1697 (MEXU); Rzedowski-Rotter 15614 (MEXU); Téllez-Valdés 9797 (MEXU); Téllez-Valdés 10134 (MEXU); Tenorio-Lezama 16643 (MEXU); Tenorio-Lezama 16649 (MEXU)].

Vernonia salicifolia (DC.) Sch. Bip. [Ramírez-Rodríguez 853 (MEXU); Téllez-Valdés 11210 (MEXU)]. 
Vernonia serratuloides Kunth subsp. serratuloides [Boutin 2091 (MEXU); Flores-Franco 2425 (MEXU); Ramírez-Rodríguez 766 (MEXU)].

Vernonia sinclairii Benth. [Téllez-Valdés 9462 (MEXU); Téllez-Valdés 10005 (MEXU); TéllezValdés 12578 (ENCB, MEXU, MICH, MO, XAL)].

Vernonia steetzii (Sch. Bip.) Gleason [Flores-Franco 2430 (MEXU); Ramírez-Rodríguez 775 (MEXU); Téllez-Valdés 11423 (MEXU); Téllez-Valdés 12469 (MEXU); Tenorio-Lezama 16721 (MEXU)].

Vernonia triflosculosa Kunth var. triflosculosa [Flores-Franco 894 (MEXU); Jones 22531 (MEXU); Téllez-Valdés 9368 (MEXU); Téllez-Valdés 10267 (MEXU)].

Vernonia triflosculosa Kunth var. palmeri (Rose) S.B. Jones [Palmer 1872 (F, GH, MICH, US)].

Viguiera angustifolia (Hook. \& Arn.) S.F. Blake [Benítez-Paredes 3389 (MEXU); FloresFranco 3186 (ENCB, MEXU, MICH, MO); Flores-Franco 3294 (MEXU); Téllez-Valdés 9415 (MEXU); Téllez-Valdés 10453 (MEXU)].

Viguiera buddleiiformis (DC.) Benth. \& Hook. f. ex Hemsl. [Téllez-Valdés 10016 (MEXU)]. Viguiera cordata (Hook. \& Arn.) D’Arcy var. cordata [Flores-Franco 1328 (MEXU); Flores-

Franco 1500 (MEXU); Flores-Franco 3131 (MEXU); González-Flores 672 (MEXU, UAN); McVaugh 16599 (MEXU, MICH); Téllez-Valdés 9373 (MEXU); Téllez-Valdés 9871 (MEXU); Téllez-Valdés 10019 (MEXU); Téllez-Valdés 11269 (MEXU); Téllez-Valdés 12987 (ENCB, MEXU, MICH, MO, US, XAL); Tenorio-Lezama 16555 (MEXU)].

Viguiera cordata (Hook. \& Arn.) D'Arcy var. websteri (B.L. Turner) McVaugh [Calzada 19066 (MEXU); Calzada 19236 (BM, EBCH, ENCB, FCME, MEXU, MICH, MO, US, XAL); Téllez-Valdés 10946 (MEXU)].

Viguiera dentata (Cav.) Spreng. var. dentata [Arcadia-Alvarez 197 (MEXU); Benítez-Paredes 3706 (MEXU); Flores-Franco 1638 (MEXU); Flores-Franco 2066 (MEXU); Flores-Franco 2373 (MEXU); Flores-Franco 2400 (MEXU); Flores-Franco 2407 (MEXU); RamírezRodríguez 717 (MEXU); Ramírez-Rodríguez 752 (MEXU); Téllez-Valdés 11498 (MEXU)]. Viguiera ensifolia (Sch. Bip.) S.F. Blake [Flores-Franco 3268 (MEXU); González-Flores 707 (MEXU)].

Viguiera flava (Hemsl.) S.F. Blake var. flava [Flores-Franco 3752 (MEXU)].

Viguiera hemsleyana S.F. Blake var. hemsleyana [Paray s.n. (MEXU)].

Viguiera huajicoria B.L. Turner [Solís 556 (TEX, CIIDIR)].

Viguiera hypochlora (S.F. Blake) S.F. Blake [Tenorio-Lezama 15680 (MEXU)].

Viguiera kingii McVaugh [King 3675 (MICH); McVaugh 16599 (MICH)].

Viguiera latibracteata (Hemsl.) S.F. Blake [Flores-Franco 1458 (MEXU)].

Viguiera microcephala Greenm. [Breedlove 44337 (MEXU)].

Viguiera pachycephala (DC.) Hemsl. var. pachycephala [Mexia 894 (Citado por McVaugh, 1984)].

Viguiera parkinsonii (Hemsl.) S. F. Blake [Breedlove 44584 (MEXU); Calzada 18847 (MEXU);

Téllez-Valdés 12402 (ENCB, MEXU, MICH, MO, XAL); Tenorio-Lezama 16024 (MEXU)]. Viguiera pringlei B.L. Rob. \& Greenm. [Flores-Franco 1903 (MEXU); Flores-Franco 2537

(MEXU); Flores-Franco 2538 (MEXU); Téllez-Valdés 10064 (MEXU); Téllez-Valdés 10074 (MEXU)].

Viguiera quinqueradiata (Cav.) A. Gray [Téllez-Valdés 11045 (MEXU)].

Viguiera schultzii S.F. Blake [Stuessy 4117 (MEXU)]. 
Viguiera stenoloba S.F. Blake [Flores-Franco 1300 (MEXU)].

Viguiera tenuis A. Gray [Flores-Franco 1657 (MEXU); Téllez-Valdés 12435 (MEXU)].

Wamalchitamia strigosa (DC.) Strother [Calzada 18695 (MEXU)].

Wedelia acapulcensis Kunth var. acapulcensis [Arcadia-Alvarez 175 (MEXU); ArcadiaAlvarez 200 (MEXU); Benítez-Paredes 3002 (MEXU); Benítez-Paredes 3182 (MEXU); Benítez-Paredes 3828 (MEXU); Flores-Franco 2696 (MEXU, MICH); Flores-Franco 3684 (MEXU); Téllez-Valdés 10675 (MEXU); Téllez-Valdés 12763 (MEXU); Tenorio-Lezama 16221 (MEXU, RSA); Tenorio-Lezama 16448 (MEXU)].

Wedelia acapulcensis Kunth var. hispida (Kunth) Strother [Benítez-Paredes 3964 (MEXU)]. Wedelia aggregata (Greenm.) B.L. Turner [Tenorio-Lezama 16779 (MEXU)].

Wedelia grayi McVaugh [Norris 14238 (MEXU, MICH)].

Wedelia mexicana (Sch. Bip.) McVaugh [Benítez-Paredes 3182 (MEXU); Flores-Franco 1405 (MEXU, MICH); Tenorio-Lezama 15943 (MEXU)].

Wedelia rosei (Greenm.) McVaugh [Flores-Franco 1423 (MEXU); Flores-Franco 2216 (ENCB, MEXU, MICH, MO); Flores-Franco 2451 (MEXU); Ramírez-Rodríguez 799 (MEXU); Ramírez-Rodríguez 946 (MEXU); Tenorio-Lezama 15936 (MEXU, RSA); Tenorio-Lezama 16541 (MEXU)].

Wedelia simsioides McVaugh [McVaugh 18735 (MICH)].

Wedelia tegetis Strother [Breedlove 45570 (CAS); Breedlove 61437 (CAS); Norris 14238 (MICH); Norris 14699 (MICH)].

Wedelia vexata Strother [King 3700 (MEXU)].

Xanthium strumarium L. [Téllez-Valdés 10352 (MEXU)].

Zinnia americana (Mill.) Olorode \& A.M. Torres [Benítez-Paredes 3411 (MEXU); Calzada 18722 (ENCB, MEXU, MICH, US, XAL); Flores-Franco 2649 (ENCB, MEXU, MICH, MO); Ramírez-Rodríguez 554 (ENCB, MEXU, MICH, MO); Téllez-Valdés 10736 (ENCB, MEXU, MICH, MO)].

Zinnia angustifolia Kunth var. angustifolia [Arcadia-Alvarez 204 (MEXU); Benítez-Paredes 3389 (MEXU); Benítez-Paredes 3414 (MEXU); Bravo-Bolaños 2132 (MEXU); Breedlove 44457 (MEXU); Calzada 18663 (MEXU, XAL); Calzada 18841 (MEXU, MICH, XAL); Campos-Villanueva 5026 (MEXU, XAL); Campos-Villanueva 5034 (MEXU, MICH, XAL); Colunga 31 (MEXU); Cronquist 9595 (MEXU); Flores-Franco 1588 (MEXU); FloresFranco 2638 (ENCB, MEXU, MICH, MO); Flores-Franco 2753 (MEXU); Flores-Franco 4014 (MEXU); García-Pérez 717 (MEXU); García-Pérez 916 (MEXU); King 3672 (MEXU); Martínez-Esquivel 705 (MEXU); Ramírez-Rodríguez 529 (ENCB, MEXU, MICH, MO, XAL); Ramírez-Rodríguez 800 (ENCB, MEXU, MICH, MO); Rzedowski-Rotter 14320 (MEXU); Stuessy 3067 (MEXU); Téllez-Valdés 9004 (MEXU); Téllez-Valdés 9063 (MEXU); Téllez-Valdés 11378 (MEXU); Téllez-Valdés 11472 (MEXU); Téllez-Valdés 12426 (MEXU); Téllez-Valdés 12980 (MEXU, MICH, XAL); Villaseñor-Ríos 711 (MEXU)].

Zinnia angustifolia Kunth var. greggii (B.L. Rob. \& Greenm.) McVaugh [Miller 3220 (MEXU, MO); Ramírez-Rodríguez 607 (ENCB, MEXU, MICH, MO, US, XAL); Ramírez-Rodríguez 650 (ENCB, MEXU, MICH, MO, XAL); Soule 2703 (MEXU, TEX); Téllez-Valdés 10554 (MEXU); Tenorio-Lezama 16928 (ENCB, MEXU, MICH); Webster 17081 (MEXU)]. 
Zinnia bicolor (DC.) Hemsl. [Benítez-Paredes 3236 (MEXU); Benítez-Paredes 3295 (MEXU); Benítez-Paredes 3606 (MEXU); Benítez-Paredes 3905 (MEXU); Flores-Franco 986 (MEXU); Flores-Franco 1454 (ENCB, MEXU, MICH, MO); Flores-Franco 2939 (ENCB, MEXU, MICH, MO, XAL); Salinas-Tovar 5126 (MEXU); Téllez-Valdés 12062 (MEXU); Téllez-Valdés 12066 (MEXU); Tenorio-Lezama 16521 (ENCB, MEXU, MICH); TenorioLezama 16738 (MEXU); Benítez-Paredes 3304 (MEXU)].

Zinnia haageana Regel [Flores-Franco 2706 (ENCB, MEXU, MICH, US)].

Zinnia leucoglossa S.F. Blake [Benítez-Paredes 3515 (MEXU); Benítez-Paredes 3522 (MEXU); Benítez-Paredes 3954 (MEXU); Rzedowski-Rotter 14318 (MEXU); Stuessy 3066 (MEXU)].

Zinnia maritima Kunth var. maritima [Howell 10374 (Citado por McVaugh, 1984)].

Zinnia peruviana (L.) L. [Tenorio-Lezama 16069 (MEXU)].

Zinnia violacea Cav. [Benítez-Paredes 3637 (MEXU); Colunga 33 (MEXU); Flores-Franco 2125 (MEXU); Salinas-Tovar 5125 (MEXU); Villaseñor-Ríos 708 (MEXU); Villaseñor-Ríos 716 (MEXU)].

Zinnia zinnioides (Kunth) Olorode \& A.M. Torres [Flores-Franco 952 (MEXU); Rose 3302 (MEXU)].

Las siguientes especies no se han registrado de Nayarit, pero se sospecha de su presencia en virtud de que se distribuyen en los estados vecinos señalados.

Ageratina mairetiana (DC.) R.M. King \& H. Rob. var. elucens (McVaugh) B.L. Turner [Durango, Jalisco, Sinaloa]

Aster moranensis Kunth var. moranensis [Durango, Jalisco, Sinaloa]

Eupatorium sonorae A. Gray [Durango, Jalisco, Sinaloa]

Flaveria trinervia (Spreng.) C. Mohr [Durango, Jalisco, Zacatecas]

Heterosperma pinnatum Cav. [Durango, Jalisco, Sinaloa, Zacatecas]

Lagascea decipiens Hemsl. var. decipiens [Jalisco, Sinaloa]

Lagascea decipiens Hemsl. var. glandulosa (Fernald) Stuessy [Jalisco, Durango, Sinaloa] Leibnitzia seemannii (Sch. Bip.) G.L. Nesom [Durango, Jalisco, Zacatecas]

Pectis linifolia L. var. linifolia [Durango, Jalisco]

Psacalium pachyphyllum (Sch. Bip.) H. Rob. \& Brettell [Durango, Jalisco, Sinaloa, Zacatecas]

Pseudognaphalium inornatum (DC.) A. Anderb. [Durango, Jalisco, Sinaloa, Zacatecas]

Stevia trifida Lag. [Durango, Jalisco, Sinaloa]

Tagetes micrantha Cav. [Durango, Jalisco, Zacatecas]

Tithonia calva Sch. Bip. var. calva [Durango, Jalisco, Sinaloa]

Verbesina cinerascens B.L. Rob. \& Greenm. [Jalisco, Sinaloa]

Vernonia barbinervis (Sch. Bip.) Gleason [Durango, Sinaloa, Zacatecas]

Zinnia grandiflora Nutt. [Durango, Zacatecas] 